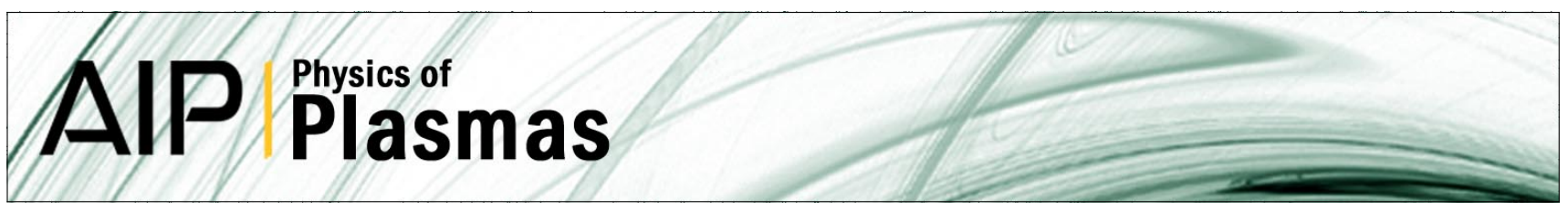

\title{
Nonlinear beam generated plasma waves as a source for enhanced plasma and ion acoustic lines
}

L. K. S. Daldorff, H. L. Pécseli, J. K. Trulsen, M. I. Ulriksen, B. Eliasson et al.

Citation: Phys. Plasmas 18, 052107 (2011); doi: 10.1063/1.3582084

View online: http://dx.doi.org/10.1063/1.3582084

View Table of Contents: http://pop.aip.org/resource/1/PHPAEN/v18/i5

Published by the AIP Publishing LLC.

\section{Additional information on Phys. Plasmas}

Journal Homepage: http://pop.aip.org/

Journal Information: http://pop.aip.org/about/about_the_journal

Top downloads: http://pop.aip.org/features/most_downloaded

Information for Authors: http://pop.aip.org/authors

\section{ADVERTISEMENT}

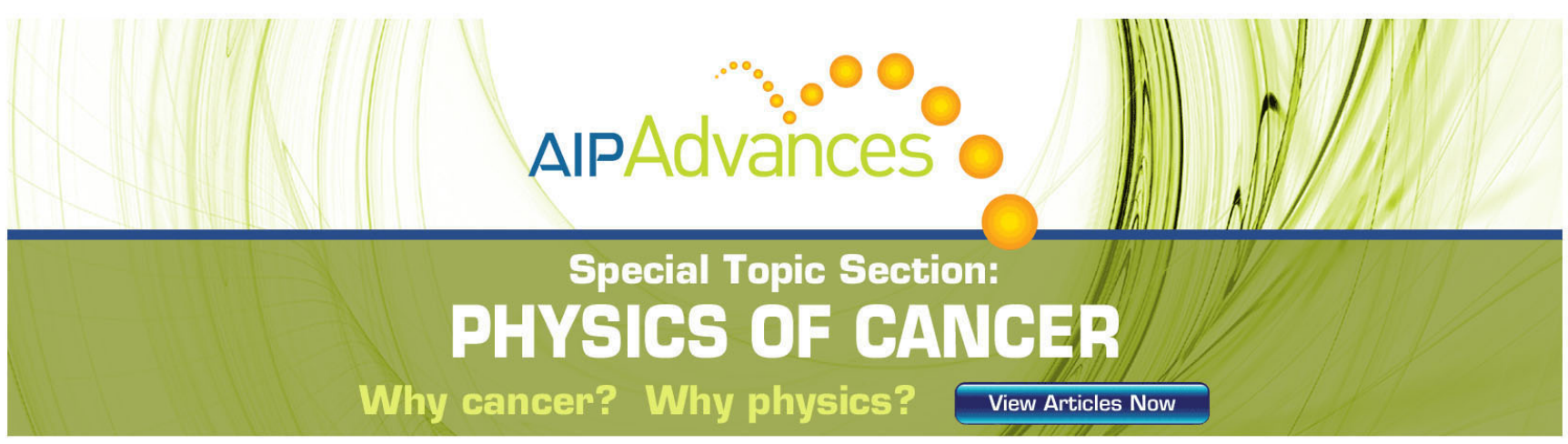




\title{
Nonlinear beam generated plasma waves as a source for enhanced plasma and ion acoustic lines
}

\author{
L. K. S. Daldorff, ${ }^{1, a)}$ H. L. Pécseli, ${ }^{2, b)}$ J. K. Trulsen, ${ }^{3, c)}$ M. I. Ulriksen, ${ }^{4, d)}$ B. Eliasson, ${ }^{5, e)}$ \\ and L. Stenflo,f) \\ ${ }^{1}$ University of Michigan, Space Research Building, 2455 Hayward Street, Ann Arbor, \\ Michigan 48109-2143, USA \\ ${ }^{2}$ Department of Physics, University of Oslo, Box 1048 Blindern, N-0316 Oslo, Norway \\ ${ }^{3}$ Institute of Theoretical Astrophysics, University of Oslo, Box 1029 Blindern, N-0315 Oslo, Norway \\ ${ }^{4}$ Norwegian Water Resources and Energy Directorate, Drammensveien 211, Postboks 5091 Majorstua, \\ N-0301 Oslo, Norway \\ ${ }^{5}$ Fakultät für Physik und Astronomie, Ruhr-Universität Bochum, D-44780 Bochum, Germany \\ ${ }^{6}$ Department of Physics, Linköping University, SE-58183 Linköping, Sweden
}

(Received 21 December 2010; accepted 1 April 2011; published online 27 May 2011)

Observations by, for instance, the EISCAT Svalbard Radar (ESR) demonstrate that the symmetry of the naturally occurring ion line in the polar ionosphere can be broken by an enhanced, nonthermal, level of fluctuations (naturally enhanced ion-acoustic lines, NEIALs). It was in many cases found that the entire ion spectrum can be distorted, also with the appearance of a third line, corresponding to a propagation velocity significantly slower than the ion acoustic sound speed. It has been argued that selective decay of beam excited primary Langmuir waves can explain some phenomena similar to those observed. We consider a related model, suggesting that a primary nonlinear process can be an oscillating two-stream instability, generating a forced low frequency mode that does not obey any ion sound dispersion relation. At later times, the decay of Langmuir waves can give rise also to enhanced asymmetric ion lines. The analysis is based on numerical results, where the initial Langmuir waves are excited by a cold dilute electron beam. By this numerical approach, we can detect fine details of the physical processes, in particular, demonstrate a strong space-time intermittency of the electron waves in agreement with observations. Our code solves the full Vlasov equation for electrons and ions, with the dynamics coupled through the electrostatic field derived from Poisson's equation. The analysis distinguishes the dynamics of the background and beam electrons. This distinction simplifies the analysis for the formulation of the weakly nonlinear analytical model for the oscillating two-stream instability. The results have general applications beyond their relevance for the ionospheric observations. (C) 2011 American Institute of Physics. [doi:10.1063/1.3582084]

\section{INTRODUCTION}

Incoherent scatter radars are some of the most versatile and widely used tools for studying the Earth's ionosphere. For the case where the ionospheric plasma is in thermal equilibrium, the backscattered signal can be analyzed in terms of the fluctuations-dissipation theorem from basic thermodynamics and statistical mechanics, ${ }^{1,2}$ giving both the ionacoustic and the electron plasma wave spectra. In many cases it is found, however, that the ionospheric plasma is out of equilibrium, and that particularly the ion-line signal is significantly distorted, ${ }^{3-5}$ giving rise to so-called naturally enhanced ion-acoustic lines (NEIALs). In the NEIALs, the two ion-lines will often have different amplitudes and correspond to velocities, which do not match the expected ion

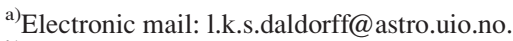

${ }^{b)}$ Electronic mail: hans.pecseli@fys.uio.no.

${ }^{c)}$ Electronic mail: j.k.trulsen@astro.uio.no.

d)Electronic mail: miul@nve.no.

${ }^{\text {e)} E l e c t r o n i c ~ m a i l: ~ b e n g t @ t p 4 . r u h r-u n i-b o c h u m . d e . ~}$

${ }^{\mathrm{f}}$ Electronic mail: lennart.stenflo@physics.umu.se. Also at Department of Physics, Umeå University, SE-901 87 Umeå, Sweden.
}

acoustic sound speed. In many cases also an unshifted ion line can be observed ${ }^{1,5}$ between the up- and down-shifted lines. These weakly shifted lines are often sporadic and can be difficult to identify, and can appear more like a "fillingin" between the two natural ion lines. Several models were suggested, ${ }^{6,7}$ and they can account for some of these features. For instance, the symmetry of the natural ion-line is broken if a current is flowing through the plasma. ${ }^{1}$ It has also been pointed out that an external "pump wave" could give effects similar to those observed. 8,9 An electron beam can enhance electron plasma waves (Langmuir waves) significantly above the thermal level, and then ion acoustic waves can be excited by parametric decay of these waves. This latter model was invoked in other studies ${ }^{10,11}$ and has gained confidence by several works. ${ }^{1,4,12}$ Consistent with the basic features of these proposed models, observations of simultaneously enhanced levels of ion, and electron plasma waves have been reported. ${ }^{13}$ The nonshifted (or weakly shifted) ion line can be explained by two basically different models. Since the speed of propagation is significantly lower than the sound speed, the line is not a natural fluid mode. It is then either a feature which has to be continuously maintained by 
some external agency, for instance, the electron beam, or, alternatively, it is a natural mode existing beyond a standard fluid model, a linear kinetic van Kampen-Case mode, ${ }^{14}$ or a nonlinear BGK-mode. ${ }^{15,16}$ Either of these modes can in principle have any velocity. In reality, the velocities will be restricted to the range of thermal velocities of the appropriate species. Propagation velocities that differ significantly from the natural sound speed require very "artificial" shapes of the velocity distributions. Some parametrized models suggested in the literature ${ }^{16}$ represent one way of imposing conditions on the distribution functions, in order to make them match physically realistic velocity distributions at large distances from the structures.

One problem concerning the model based on Langmuir wave decay seems to be that sometimes very short wavelength primary Langmuir waves are needed to account for the observations, below a few tens of Debye lengths, $\lambda_{D e}$. It might be possible to find a low velocity electron beam which generates unstable waves for $u_{b}<4 u_{t h}$, but the decay Langmuir wave ("daughter wave") obtained from these will be strongly Landau damped, implying that the growth rate of the decay instability becomes negligible. For the EISCAT Svalbard Radar (ESR)-radar, ${ }^{3}$ we have a transmitter frequency of $500 \mathrm{MHz}$, giving $\lambda_{R} \equiv 2 \pi / k_{R}=0.6 \mathrm{~m}$. For an altitude $\sim 400 \mathrm{~km}$, with electron temperatures of $T_{e} \approx 3000$ $\mathrm{K}$, and plasma densities of $n_{0} \approx 2 \times 10^{11} \mathrm{~m}^{-3}$, we find the Debye length $\lambda_{D e} \approx 8.5 \times 10^{-3} \mathrm{~m}$, i.e., $\lambda_{R} / \lambda_{D e} \sim 70$, or $k_{R} \lambda_{D e} \approx 0.09$. The effects connected with plasma inhomogeneity have been largely ignored, although a consistent treatment of the ionospheric plasma density gradient can bring new understanding of the observational results. ${ }^{17,18}$

The ionospheric observations as such seem to be unambiguous, ${ }^{3}$ but the interpretation is made difficult by several practical problems: the observed features are often sporadic in nature, and can vary with time as well as altitude, on second and kilometer scales, respectively. ${ }^{1,12} \mathrm{We}$ bear in mind that the radar is usually obtaining backscatter at one selected wavevector $\mathbf{k}_{R}$, which is related to the scattering wavevector $\mathbf{k}_{B}$ by selection rules, which for a monostatic radar gives $\mathbf{k}_{B}=2 \mathbf{k}_{R}$. In order to observe the ion sound wave, we should have the sound wavelength $\lambda_{s}$ approximately equal to $(1 / 2) \lambda_{R}$ and the primary Langmuir wavelength $\lambda_{L}$ also approximately $(1 / 2) \lambda_{R}$. This means that in a decay process, we cannot, usually, at a given altitude, expect to observe the first generation Langmuir waves simultaneously with the sound waves forming the low frequency part of the decay products. In the case where we have a "cascade" of decaying waves, we might observe one or the other of the decay products, and it might very well be the second or third generation that is observed, instead of the first one. For the parameters mentioned before, we have $k_{L} \lambda_{D e} \approx 0.18$. The plasma conditions are strongly variable $^{19}$ and it is not always obvious under which conditions the enhanced ion-lines (NEIALs) are observed, since the relevant parameters are rarely monitored simultaneously. The observations are not sufficiently detailed to allow only one model for their explanation. It has been $\operatorname{argued}^{13}$ that a broad band of Langmuir waves excited by an electron beam with distributed velocities $\left(\Delta u_{b} \sim u_{b}\right)$ can generate a wide spectrum of waves that can account for the simultaneous observa- tions of enhanced ion and electron lines at the same Bragg condition, but these calculations have seemingly not been published nor tested by numerical simulations.

The simple Langmuir wave decay can seemingly account for the enhanced ion lines, but cannot directly explain the unshifted component. In our first attempt to explain this feature we considered the possibility of excitation of ion phase-space vortices, or ion holes. These structures are well known from laboratory experiments and numerical simulations. ${ }^{20,21}$ It has been found that excitation of ion holes is ineffective when the electron/ion temperature ratio is below two, ${ }^{20}$ and this is after all the most common parameter range for many ionospheric conditions. Analytical and numerical studies ${ }^{22}$ have demonstrated that ion phase space holes can be maintained by an enhanced level of Langmuir waves even for moderate ratios $T_{e} / T_{i}$. One purpose of the present study was to search for self-consistently and spontaneously generated ion holes with a trapped electron wave component. In order to make the conditions for ion hole formation demanding, we choose a temperature ratio of $T_{e} / T_{i}=1$. Actually, it is possible to construct ion phase-space vortices for any temperature ratio $T_{e} / T_{i}>0$, but as stated before there is empirical evidence ${ }^{20}$ that $T_{e} / T_{i} \approx 2$ is a limiting temperature ratio for their existence in practice.

Electron phase-space vortices, or electron holes, ${ }^{16,23,24}$ can be excited and they will have interest in the present context also because such structures can have, in principle, any velocity, also one below the ion sound speed. For physically realistic velocity distribution functions, electron phase-space vortices move at or below the electron thermal velocity ${ }^{25}$ (with ion vortices having velocities at or below the ion thermal velocity). Although these vortices can be slow compared to the ion sound speed, it will be unlikely to see them confined to such slow velocities. Only their ion counterparts are realistic candidates for subsonic nonlinear structures. Electron holes are well known from laboratory experiments, ${ }^{23,26}$ but their possible role in the generation of NEIALs and the intermediate slow or subsonic ion signature is unknown.

Numerical simulations offer a possibility for studying the relevant plasma phenomena in detail. ${ }^{12,27-30}$ The present study is based on a direct numerical solution of the coupled electron and ion Vlasov equations solved for a linearly unstable beam-background electron population. The analysis distinguishes, in particular, the dynamics of background and beam electrons as well as the ions.

Sections II and III present our numerical analysis. The theoretical analysis in Secs. IV and V supports the numerical results. In particular, that analysis distinguishes the dynamics of the background and the beam electrons, in agreement with the numerical results. For the formulation of the weakly nonlinear model for the oscillating two-stream instability, this distinction turns out to have interesting implications. The wave types entering the analysis are the usual Langmuir waves with dispersion relation $\omega^{2}=\omega_{p e}^{2}+3 k^{2} u_{t h}^{2}$, and electron acoustic waves $^{31,32}$ with an almost linear proportionality between frequency and wavenumber. Our analytical results support a model where electron waves are excited by the electron beam instability, with a dominant mode following an electron 
acoustic dispersion relation. Through an oscillating two-stream instability, this wave subsequently excites a broad band of Langmuir waves together with low frequency, broad band electrostatic oscillations that do not satisfy any dispersion relation. In a later stage of the wave evolution, ion acoustic waves are excited by Langmuir wave decay, where the electron acoustic mode participates.

A one-to-one numerical simulation of the problem with relevant ionospheric parameters is not possible, so the present work addresses some qualitative features that we believe to be relevant. Some previous preliminary results from related studies ${ }^{33}$ supplement the analysis presented here by using different plasma parameters.

\section{BEAM-DRIVEN ELECTRON PLASMA WAVES}

Concerning the unstable electron plasma waves, we have two limiting cases. When the beam velocity is much larger than any of the relevant thermal velocities, the most unstable phase velocity is somewhere between the beam and background plasma velocities, in a region where the electron velocity distribution is close to vanishing. For this case, we might as well assume that both the beam and background distributions are adequately represented by $\delta$-functions. In this limit, the instability can be modeled by a simple two-electron beam model. In the other limit, the phase velocity $u_{p h}=\omega / k$ of the most unstable mode will be close to the beam velocity $u_{b}$, so that $u_{b}-u_{T b} \leq u_{p h}<u_{b}$, with $u_{T b}=\sqrt{T_{e b} / m}$ being the thermal velocity of the electron beam. For this fully kinetic limit, the instability is of the standard Landau type, where the growth rate is determined by the slope of the electron velocity distribution at the phase velocity. The full time evolution can involve both models: in an initial phase, we can have the standard cold-beam model applying, until the waves have reached an amplitude where they effectively scatter the beam so that it disperses in velocity space. The resulting "plateau" in the electron velocity distribution supports an electron acoustic branch. When a significant number of particles are scattered into the velocity range of the linearly most unstable waves, the process will continue at a rate determined by the fully kinetic theory, implying a "two-stage" process of the instability. Using a double water-bag model for the plasma (one for the beam and one for the background) we obtain the linear dispersion relation

$$
\begin{aligned}
1+ & (1-\alpha)\left(\frac{\omega_{p e}}{k}\right)^{2} \frac{1}{u_{s}^{2}-(\omega / k)^{2}} \\
& +\alpha\left(\frac{\omega_{p e}}{k}\right)^{2} \frac{1}{\Delta u^{2}-\left(u_{b}-\omega / k\right)^{2}}=0,
\end{aligned}
$$

where $\alpha=n_{02} / n_{0}$ is the relative electron beam density, $u_{s}$ is the water-bag boundary velocity for the background plasma, while $\Delta u$ is the width of the beam water bag in velocity space. The background electron density is $n_{01}$, so that $n_{01}+n_{02}=n_{0}$. We recall that for the background water bag electrons we have $\sqrt{\left\langle u^{2}\right\rangle}=u_{s} / \sqrt{3}$, which is a relation needed for defining the effective background electron temperature.

The linear dispersion relation for the problem can be solved analytically (albeit with a lengthy general result) for the case where the beam and background electron temperatures are vanishing, in which case the beam velocity enters via the normalizations. ${ }^{33}$ A somewhat more general result for the reference case is shown in Fig. 1, obtained by the water-bag model (1), which corresponds to a standard two-fluid model for the electrons. ${ }^{34}$ The important part is the unstable branch, appearing as a weakly dispersive electron acoustic mode. The most unstable part of this mode appears here at frequencies below the electron plasma frequency. Finite geometry (the finite diameter of a plasma column or the width of an elongated plasma cavity), as in some experiments, ${ }^{35}$ implies nontrivial modifications of the dispersion relation.

For a wide range of parameters for cold beams with $\alpha \equiv n_{02} / n_{0} \leq 0.1$, the most unstable wave is found for $k u_{b} / \omega_{p e} \approx 1.2$. For $\alpha \rightarrow 0$, we have $k u_{b} / \omega_{p e} \rightarrow 1$. In particular, for $\alpha=1 / 2$, we find the most unstable wave for a phase velocity $u_{b} / 2$ as expected by symmetry reasons. For small $\alpha$ the most unstable phase velocity increases, ultimately to reach $u_{b}$, with the most unstable frequency $\omega \leq \omega_{p e}$. As an approximation, we have the phase velocity for the most unstable wave as $u_{p h} \approx u_{b}(1-\alpha)$. At the same time, we find that the linear growth rate of the instability decreases with decreasing $\alpha$ so that the plasma is stable in the limit where the most unstable frequency approaches $\omega_{p e}$. During this transition, the entire electron acoustic branch remains unstable, with a growth-rate proportional to $k$ in the long-wavelength limit. As an approximate criterion for the beam instability to be relevant, rather than the Landau instability, we have $u_{b}(1-\alpha)<u_{b}-u_{T b}$ or $\alpha>u_{T b} / u_{b}$. The transition from beam to Landau type instabilities were previously studied by analyzing the linear dispersion relation. ${ }^{36}$

The electron beam is exciting electron plasma waves by the beam instability. The dominant wave component will be the one with the largest temporal growth rate, which here corresponds to a frequency below $\omega_{p e}$. When these wave amplitudes reach a sufficient intensity, they can excite new waves by the oscillating two-stream instability (see, for instance, ${ }^{37}$ for an excellent summary). The low frequency component of these wave modes need not be represented by a (linear) dispersion relation and can in principle be stationary, while the high frequency wave component will have an angular frequency closer to $\omega_{p e}$.

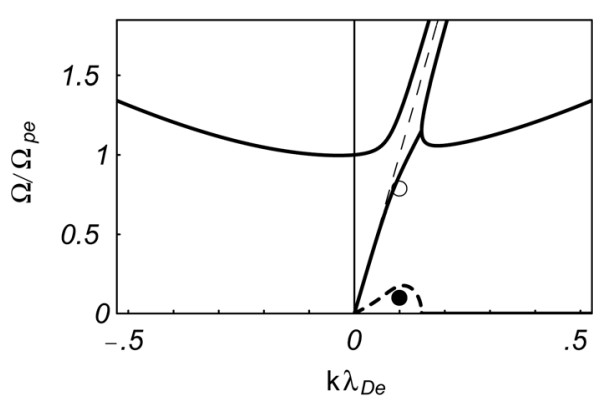

FIG. 1. Dispersion relation for the reference case with $n_{02} / n_{0}=0.025$, with the real part of the frequency $\Re\{\omega\}$ given by a thick full line, and the growth rate $\Im\{\omega\}$ given by a thick dashed line, while a thin dashed line gives the beam velocity $u_{b}$ for reference. We have $T_{e b}=T_{e} / 5$ The symbol ${ }^{\circ}$ shows the most unstable wave $(\omega, k)$ from the simulation, found in the time interval $\left\{0 ; 75 / \omega_{p e}\right\}$, and $\bullet$ gives the corresponding growth-rate observed. 


\section{NUMERICAL RESULTS}

The basic equations for the numerical simulations are the Vlasov equations for the electrons and ions, with the dynamics coupled through Poisson's equation. Details of the code have been presented elsewhere. ${ }^{22,38,39}$ The normalizations used in the code, in particular, is given by Ref. 40 . We use here a spatial simulation interval of $200 \pi \lambda_{D e}$. The basic set of equations is solved for an initial value problem with periodic spatial boundary conditions, which is standard for these types of problems. This gives a simplified alternative to the full problem with conditions imposed at a boundary, while at the same time retaining the important physics. The analysis is restricted to one spatial dimension for practical computational reasons. In particular for the case of Langmuir wave decay, we do not expect this to pose any serious limitation, in part because the growth rate for the decay instability is known to have a maximum for aligned wavevectors. ${ }^{41}$ For the observations relevant to the present study, ${ }^{4}$ the radar beam is basically directed along the magnetic field lines, inviting a comparison with models referring to that direction.

We have analyzed a large parameter range,

$$
\begin{gathered}
u_{b} / u_{T e}=2-10 . \\
n_{02} / n_{0}=0.01-0.1, \text { and } T_{e b} / T_{e 0}=0.2-0.6 .
\end{gathered}
$$

We have used representative plasma parameters, so that the numerical results can be used for qualitative and to some extent quantitative comparisons with observations. Practical limitations are, however, imposed by the numerical code. For instance, the effects have to be observable within at most a few thousand electron plasma periods. This condition restricts our mass ratio and imposes limits on the relative beam density $n_{02} / n_{0}$. In the first part of our simulations, we use an ion to electron mass ratio of $M / m=400$. In order to have a well defined electrostatic wave problem, we choose the initial condition so that there is no dc-current in the system. We let the background electron population drift slightly in the opposite direction of the beam. The code has an extremely low noise level, as compared to, for instance, standard particle-in-cell (PIC) codes. In order to achieve a noticeable amplitude of the unstable modes within a reasonable computing time, we introduce some initial irregularities by a low level density perturbation containing many Fourier components with wavelengths in the range $\{\mathcal{L} / 20 ; \mathcal{L}\}$ with $\mathcal{L}$ being the length of the system. An interesting alternative model for this initial noise level has been given by Ref. 12, who use a self-consistent representation that allows the instability to grow out of a synthetic thermal noise level. Preliminary results from related studies ${ }^{33}$ used higher beam densities and can be seen as supplements to the analysis presented here.

\section{A. The initial time evolution}

We first study, the space-time evolution of the electron beam density for the early part of the time development, $t \in\left\{0 ; 75 / \omega_{p e}\right\}$. We observe a nearly exponential growth of a spatially wide wave packet with well defined wavenumber. The growth rate of the wave can be determined with good accuracy and is inserted in Fig. 1. A broad wavenumber band is unstable, but the initial time wave development is dominated by the linearly most unstable wave, so that the evolution appears to contain only one mode of oscillations.

In Fig. 2, we show the phase-space representation of the electron beam alone, taken at time $t=75 / \omega_{p e}$, as well as the phase-space variation of the background electrons taken at the same time. The linear instability seems to saturate in a wave-packet with a soliton-like shape. ${ }^{42,43}$ This structure is not stable, but dissolves due to the phase-space breaking of the beam electrons as can be observed in Fig. 2. The breaking is seen only in the beam electrons and not in the background, since only the near-resonant particles are affected.

In Fig. 3, we show the spatial and temporal Fourier transform of the electron beam density in an $\left(\omega, k_{x}\right)$-distribution, and also the corresponding representation of the background electrons. These and similar figures are with logarithmic color scale (base 10). The temporal Fourier transform is here restricted to $t \in\left\{0 ; 75 / \omega_{p e}\right\}$. The figures possess an $\left(\omega, k_{x}\right) \rightarrow\left(-\omega,-k_{x}\right)$-symmetry, but the properties of the low
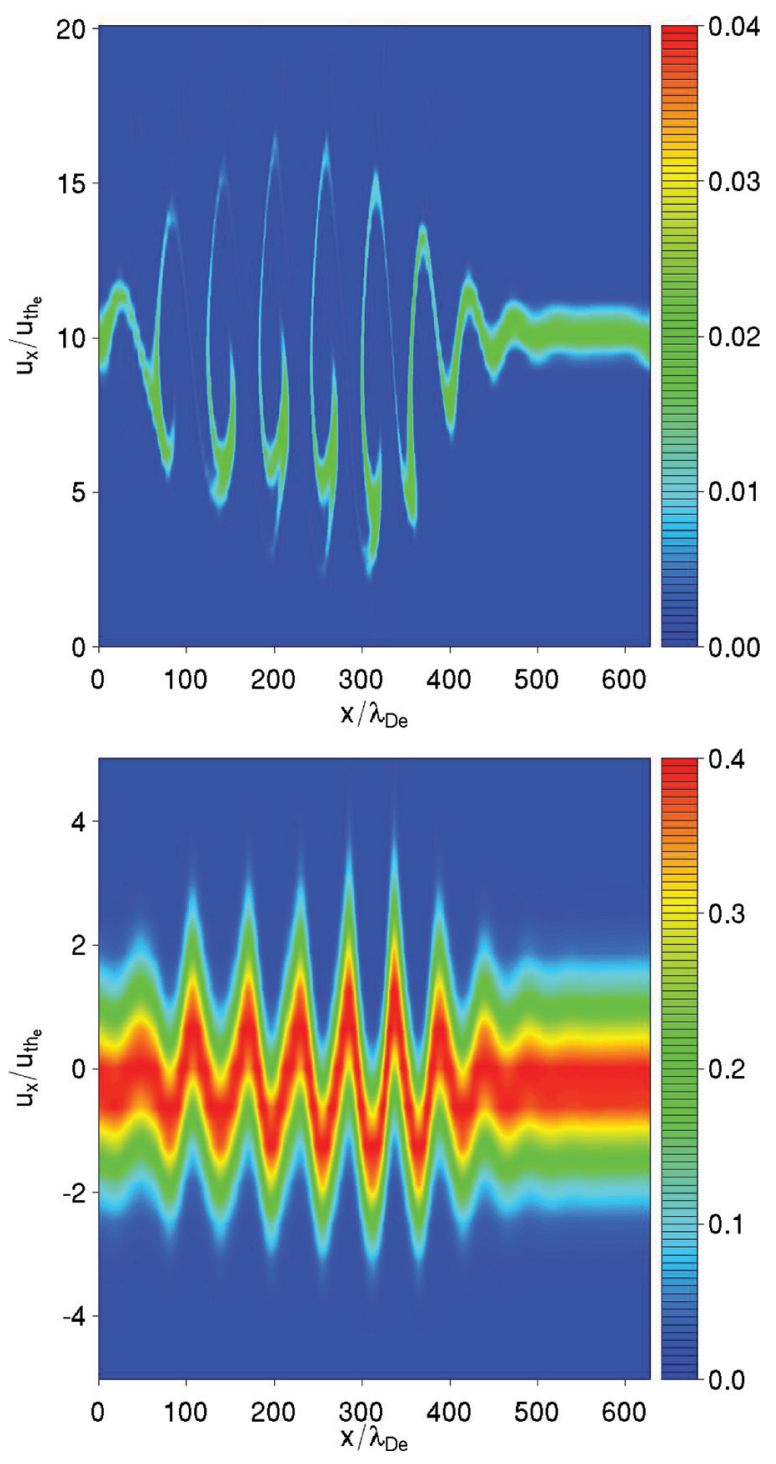

FIG. 2. (Color online) Top: phase-space distribution of the beam electrons at time $t=75 / \omega_{p e}$. Bottom: phase-space distribution of the background electrons at time $t=75 / \omega_{p e}$. Note that the color-coding has been changed by a factor 10 , as compared to the figure at the top. 

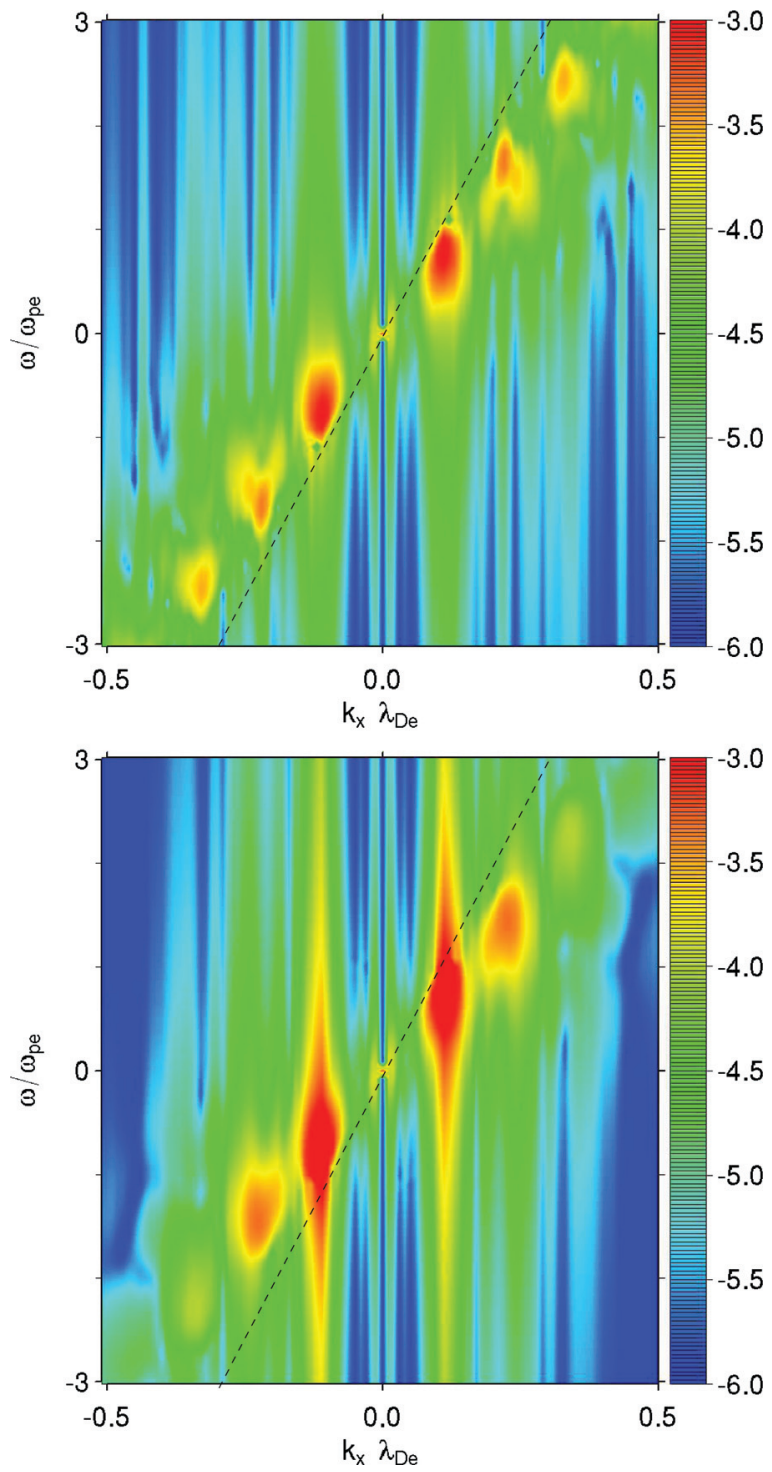

FIG. 3. (Color online) Top: temporal and spatial Fourier transforms of the beam electron density, shown in an $\left(\omega, k_{x}\right)$-plane. Colors are with logarithmic scale. A dashed line gives the electron beam velocity for reference. The analysis is restricted to the time interval $t \in\left\{0 ; 75 / \omega_{p e}\right\}$ We can readily identify the most unstable mode, which is plotted into Fig. 1. Bottom: temporal and spatial Fourier transforms of the background electron density, shown in an $\left(\omega, k_{x}\right)$-plane. A dashed line gives the electron beam velocity. The analysis is restricted to the time interval $t \in\left\{0 ; 75 / \omega_{p e}\right\}$.

frequency parts are best seen in a representation retaining this redundancy. We note a small "gap" in the $\left(\omega, k_{x}\right)$-plane around $k=0$, which is due to the finite length of the system, that places a lower limit on available wavenumbers.

We can clearly identify the real part of the frequency $\Re\{\omega\}$ as well as the wavenumber $k$ for the most unstable wave. These values are indicated in the linear dispersion relation in Fig. 1. While the fundamental wave is clearly visible in both the beam and the background dynamics, we note that the third harmonics generated by nonlinear effects are different in the two representations: for the background the third harmonic is relatively much weaker than for the beam mode because the harmonic excitations are close to resonant for the slow beam mode, in contrast to the background electrons that support a wave near the electron plasma frequency. The observed initial time evolution is in
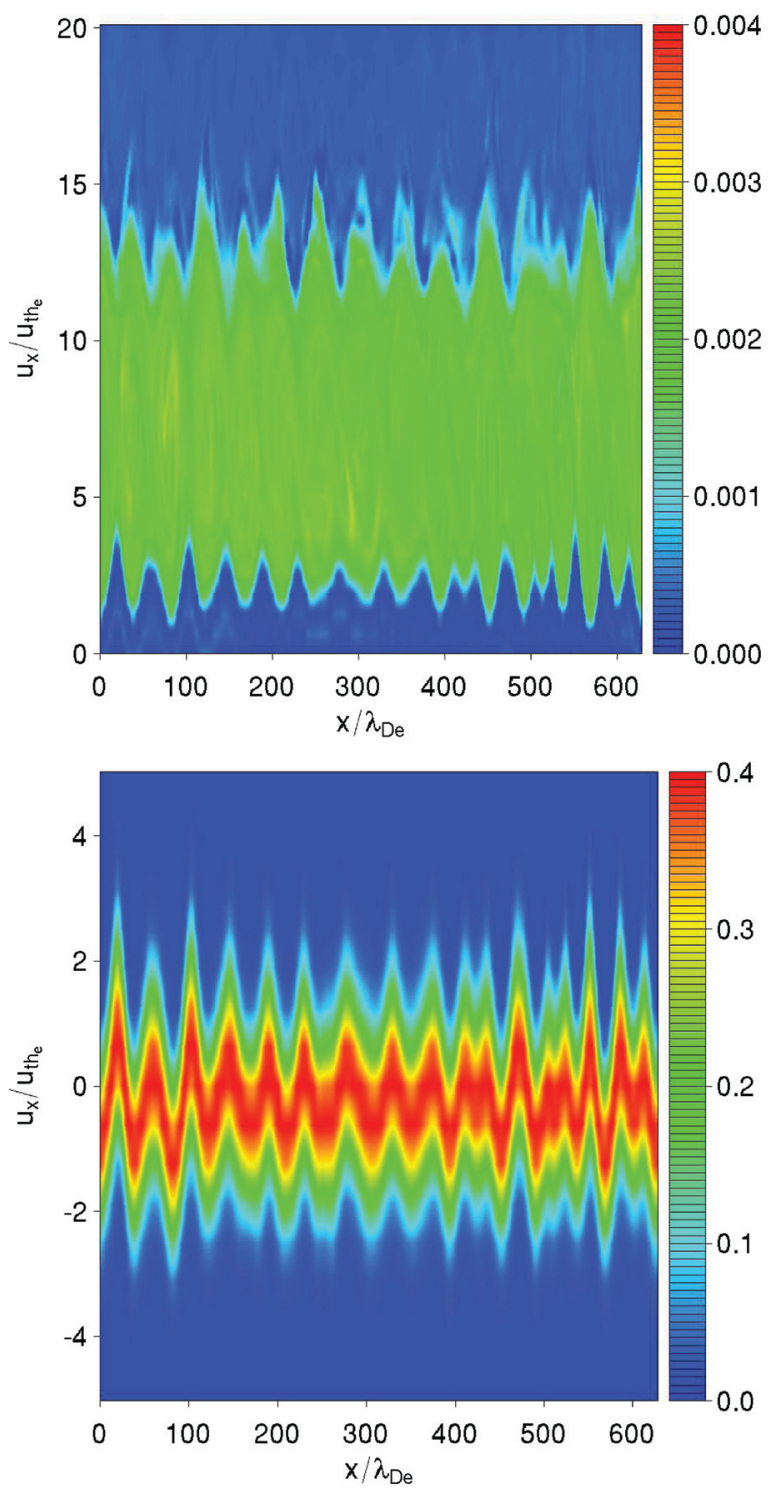

FIG. 4. (Color online) Top: phase-space distribution of the electron beam at a time $t=500 / \omega_{p e}$. Bottom: phase-space distribution of the electron background at a time $t=500 / \omega_{p e}$. Note that the color-coding has been changed by a factor 100 , as compared to the figure at the top.

good agreement also with laboratory experiments, ${ }^{44}$ but the numerical simulations can provide, for instance, phase-space information that is not available in the laboratory.

\section{B. The intermediate time evolution}

In Fig. 4, we show the phase-space distribution of the beam and the background electrons, respectively, for a later time in the evolution of the instability, here at $t=500 / \omega_{p e}$.

By inspection of Fig. 4 we find that the linear initial instability described by Fig. 1, saturates into a stage where the electron beam has spread out to form a plateau extending down to the background electron component. ${ }^{44}$ We can model the dispersion relation for this stage by keeping the original background electrons, but transform the beam into a plateau, so that the number of electrons in the beam is conserved. The plateau extends down to the boundary $u_{t h}$ of the background component. The upper boundary of the plateau is $u_{0}+(1 / 2) \Delta u$, in terms of the original beam velocity and beam width. There is now no longer any gap 


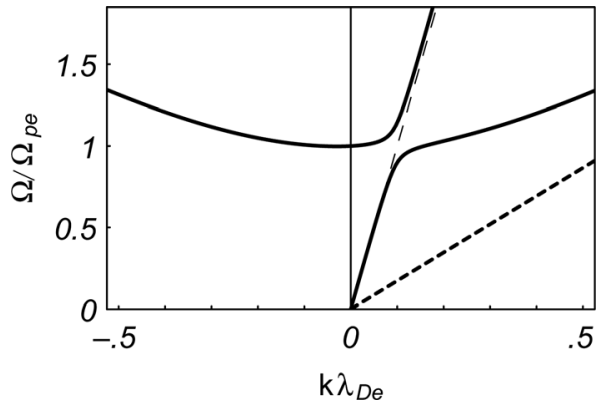

FIG. 5. Dispersion relation for the fully developed reference case resulting from the unstable condition given by Fig. 1. The electron distribution is here modeled by the same water-bag for the background electrons, while the beam has been changed into a plateau, with the same electron density as before, but with the plateau now extending to the boundary of the water-bag for the background electrons. The plasma is now stable. The thin dashed line for the original beam velocity $u_{b}$ in Fig. 1 is retained for reference.

in the electron distribution between the beam and the background components. The resulting dispersion relation shown in Fig. 5 can be obtained by redefining some of the parameters entering Eq. (1). In all cases we have $\omega=\omega_{\text {pe }}$ for $k=0$. The distribution is here linearly stable, since it now has only one local maximum. We note the presence of two electron acoustic modes, one with phase velocity close to $u_{b}$ and one where the phase velocity is approximately given by $u_{t h}$. This latter mode (shown with dashed line) is formally a solution in the present water-bag model, but it is strongly Landau damped by the Maxwellian distribution of the background electrons, and it will generally not be observed. We note that for large $|k|$ we have a part of the dispersion relation well approximated by the usual Langmuir result $\omega_{0}^{2} \approx \omega_{p e}^{2}+3 k^{2} u_{t h}^{2}$, just as in Fig. 1 .

In Fig. 6, we show the space-time Fourier transform of the electron beam and the electron background densities, respectively, for the time interval $\left\{75 / \omega_{p e} ; 500 / \omega_{p e}\right\}$. In the top Fig. 6, we recognize the electron beam modes, and note the enhancement of wave intensity around the electron plasma frequency, $\omega_{p e}$. The corresponding figure for the background electrons is entirely different. Here we note an enhancement of the wave activity for the forward propagating electron plasma wave, and some enhancement for frequencies slightly below $\omega_{p e}$, on the upper part (at the bending of the curve) of the electron acoustic wave, see the dispersion relation in Fig. 5. The backward traveling electron plasma wave mode is visible. Since the waves are enhanced to a nonthermal level, we expect second harmonics to be noticeable, as indeed observed. ${ }^{29,45,46}$

\section{A simple linearized fluid model}

A simple linear fluid model can be proposed to account for some elements of the results for times after formation of the plateau in the electron beam component

$$
\begin{gathered}
\frac{\partial^{2}}{\partial t^{2}} n_{1}-u_{t 1}^{2} \nabla^{2} n_{1}+\frac{e}{m} n_{01} \nabla^{2} \phi=0, \\
\left(\frac{\partial}{\partial t}-\mathbf{u}_{0} \cdot \nabla\right)^{2} n_{2}-u_{t 2}^{2} \nabla^{2} n_{2}+\frac{e}{m} n_{02} \nabla^{2} \phi=0,
\end{gathered}
$$

with subscripts 1 and 2 referring to the background and plateau electrons, respectively, and $\mathbf{u}_{0}$ being the average velocity of the plateau electrons.
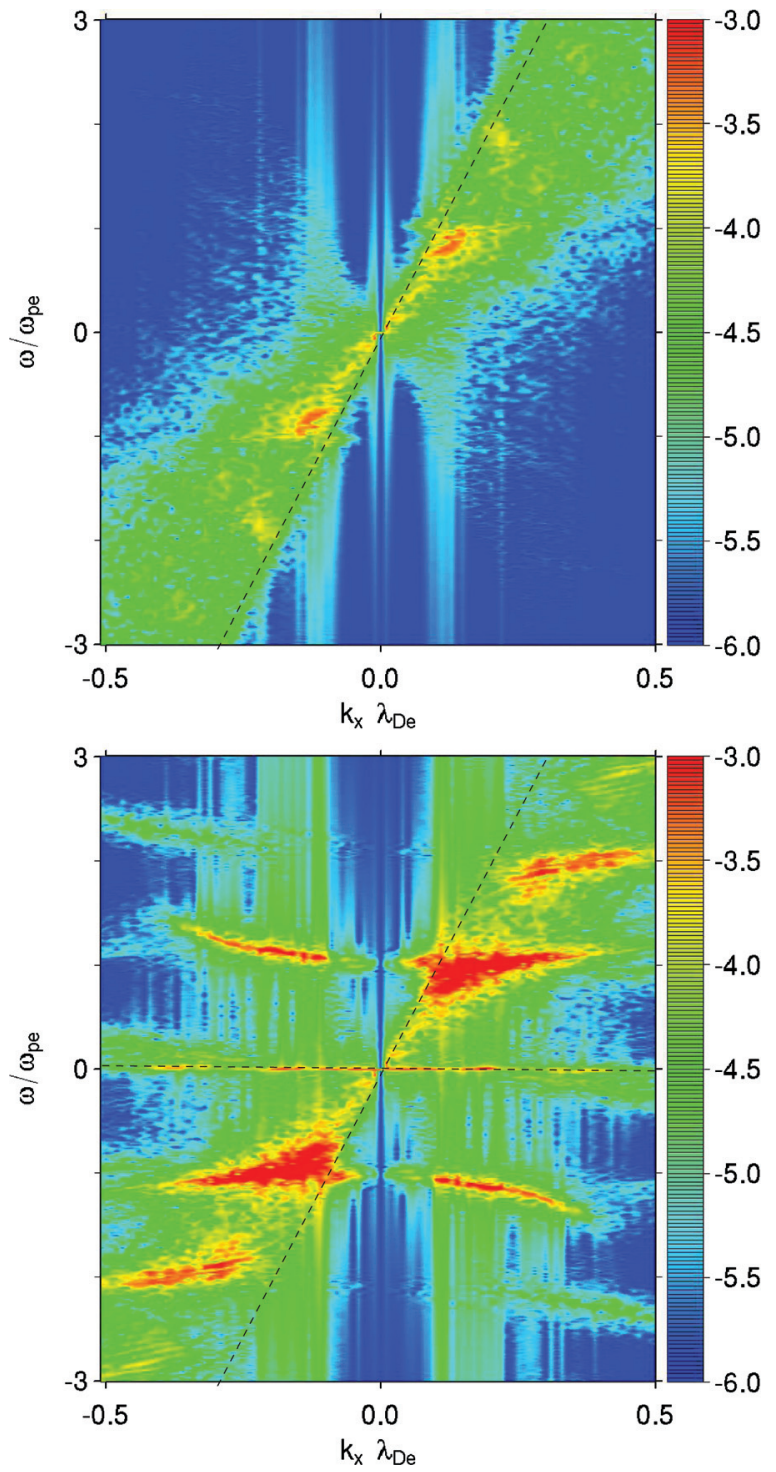

FIG. 6. (Color online) Top: temporal and spatial Fourier transforms of the beam electron density, shown in an $\left(\omega, k_{x}\right)$-plane. Colors are with logarithmic scale. A dashed line gives the original electron beam velocity. The analysis is restricted to the time interval $t \in\left\{75 / \omega_{p e} ; 500 / \omega_{p e}\right\}$. Bottom: temporal and spatial Fourier transforms of the background electron density, shown in an $\left(\omega, k_{x}\right)$-plane. Dashed lines gives the original electron beam velocity and the negative ion sound speed for reference. The analysis is restricted to the time interval $t \in\left\{75 / \omega_{p e} ; 500 / \omega_{p e}\right\}$.

The relations (2) and (3) are coupled through Poisson's equation

$$
\nabla^{2} \phi=\frac{e}{\varepsilon_{0}}\left(n_{1}+n_{2}\right) .
$$

The model is derived for a water-bag model where the background electrons have boundaries $\left\{-u_{1}, u_{1}\right\}$ and density $n_{01}$ giving $u_{t 1}=u_{1} / \sqrt{3}$, while the plateau electron component has boundaries $\left\{u_{1}, u_{b}\right\}$, and density $n_{02}$.

Numerical solutions of the coupled equations (2-4) have been carried out. For illustration, the initial perturbation was chosen to be a sinusoidal variation corresponding approximately to the linearly most unstable wavelength, $\lambda_{0}$ excited by the beaming instability. Alternatively, we used a broad spectrum obtained by a random superposition of wave-packets. ${ }^{47}$ 
Initially, we took $n_{1}=0$, i.e., no perturbation of the background electrons. Physically this corresponds to the case where the linear instability has developed and scattered the beam electrons so that they form a plateau, see Figs. 2 and 4. These electrons retain their spatial fluctuation amplitudes, while the motion of the background electrons is ignored so far. We use this state as the initial condition. At later times, both branches of the dispersion relation are excited, both with wavelength $\lambda_{0}$. The beating between these two modes gives rise to an interference pattern, where details depend on the initial distribution of wave amplitudes, but the qualitative features are independent of the initialization. The excitation of waves on the Langmuir-like branch with wavelengths corresponding to the unstable spectrum is thus not due to nonlinear effects. We found $n_{1} / n_{0}$ and $n_{2} / n_{0}$ to be of the same order of magnitude, implying that $n_{1} / n_{01} \ll n_{2} / n_{02}$, with $n_{01} \gg n_{02}$.

\section{Late times}

We studied the space-time evolution of the ion density in the time interval $t \in\left\{0 ; 800 / \omega_{p e}\right\}$ and noted the evolution of narrow, localized spikes and depletions having distributed propagation velocities. The space-time Fourier transform of the ion density is shown in Fig. 7. We note a dominant component around small wavenumbers, seemingly with a propagation velocity below the ion sound speed. We note the presence of a backward traveling ion sound component, but its amplitude is small at this time.

We have also carried out simulations with 50\% longer time duration and a somewhat reduced mass ratio $M / m=200$ to include more ion plasma periods. As expected we see no differences at all in the initial evolution, as summarized here in Figs. 2 and 3. In this time interval the ions have no time to move, due to their large inertia. Significant differences between the two simulations are found only very late in the time evolution. We show the temporal and spatial Fourier transforms of the ion density in an $\left(\omega, k_{x}\right)$-plane in the bottom Fig. 7. It shows a pronounced increase in the ion activity at these late times. The predominant feature is an increased level of backward propagating ion sound waves with relatively large wavenumbers, up to $k \lambda_{D e} \approx 0.5$, and large bandwidths. We note a significant line-broadening and a banded structure of the wave spectrum. In addition, we find an enhancement of the long-wavelength component, which was seen already in the top Fig. 7. This latter part corresponds to forward propagating waves. The corresponding analysis of the electron component is not shown here. These results look very much like those in Fig. 6, except for an additional enhancement of the backward traveling electron plasma mode around $\omega_{p e}$. We note the banded structure of the backward traveling waves. The first band is centered at a wavenumber which is close to the wavenumber separation appearing as a band between the forward and backward traveling electron plasma waves in Fig. 6. The slope of the small "glitches" in the ion sound spectrum corresponds quite accurately to the electron beam velocity. In multidimensional plasma simulations long term variations can be expected ${ }^{48}$ but these phenomena will not be seen here.

Our conclusion from the analysis summarized in Secs. III A and III B is that the initial part of the time evolution is
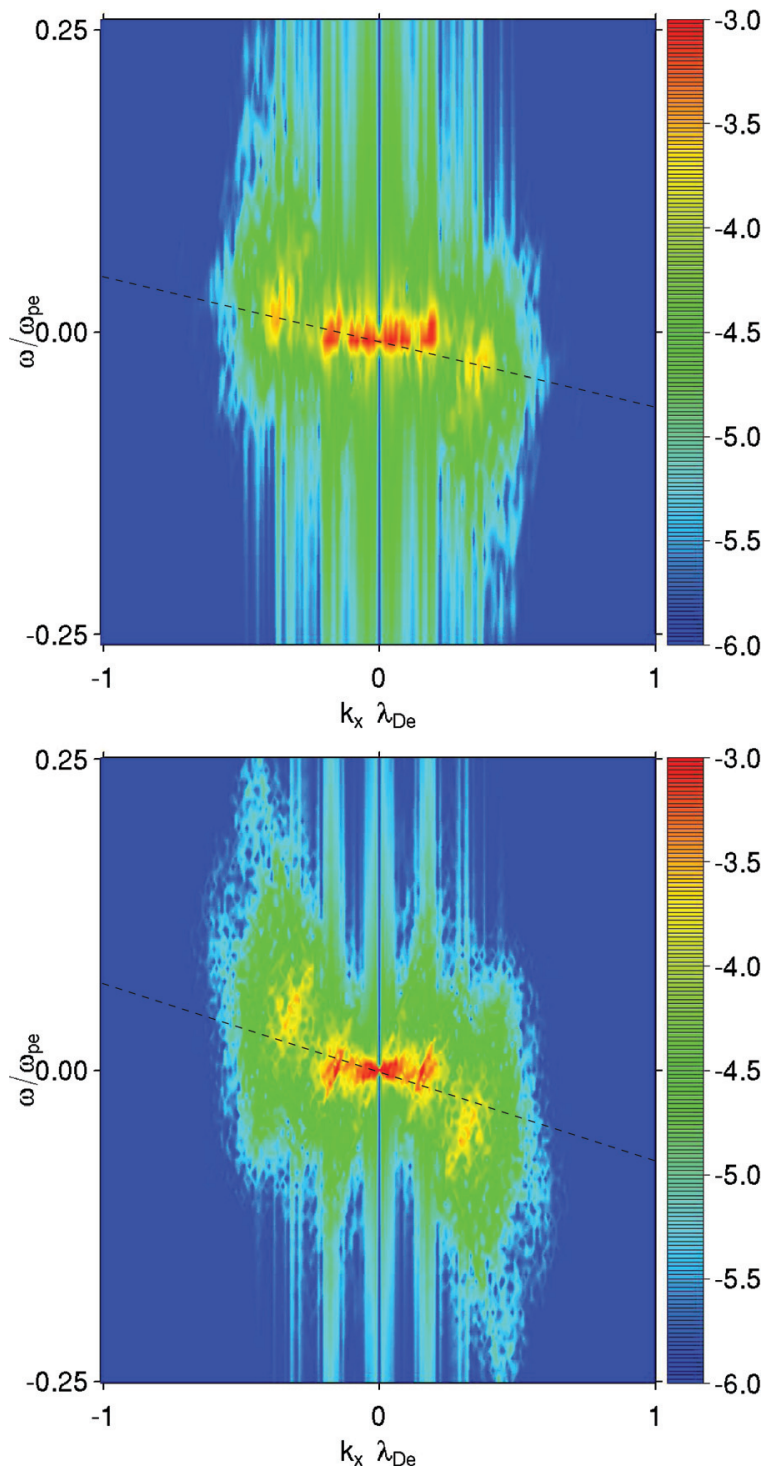

FIG. 7. (Color online) Top: temporal and spatial Fourier transforms of the ion density, shown in an $\left(\omega, k_{x}\right)$-plane, see also Fig. 6 . The dashed line with a negative slope gives the sound speed. The analysis is restricted to the time interval $t \in\left\{75 / \omega_{p e} ; 500 / \omega_{p e}\right\}$. Bottom: temporal and spatial Fourier transforms of the ion density, here with $M / m=200$, shown in an $\left(\omega, k_{x}\right)$-plane. The dashed line with a negative slope gives the ion sound speed for the present mass ratio. The analysis is here covering a time interval $t \in\left\{75 / \omega_{p e} ; 1300 / \omega_{p e}\right\}$.

essentially described by the (unstable) linear electron beam dispersion relation. The enhanced wave amplitudes disperse the beam basically by electron trapping in the dominant wave component (see Fig. 2) rather than quasilinear phasespace diffusion. (The quasilinear phase-space diffusion is found only for initially broad "bump-on-tail" distributions.) A quasistable plateau is formed by this process. The distribution function is stable and supports an enhanced fluctuation level of electron waves. The space-time Fourier transform allows the dispersion branches to be identified, and these are well accounted for by the dispersion relation obtained by a simplified water-bag model, where an electron acoustic mode enters as an important element. At the time this dispersion relation is established, we find that a broad range of wavenumbers have been populated on the Langmuir-mode like dispersion branch, see Fig. 6 . At the same time we note 
a corresponding broad wavenumber range of low frequency wave enhancements. These do not seem to fall on any dispersion relation, where ion acoustic waves would have been a relevant candidate. At a much later stage, we do find ion acoustic waves excited, but these have a banded structure and seem to be predominantly backward propagating. Reducing the electron-ion mass ratio (as in Fig. 7) allows this latter process to be observed at an earlier time.

The dominant mode observed is for frequencies and wavenumbers close to the linearly most unstable waves $\left(\omega_{0}, k_{0}\right)$, consistent with the analytical dispersion relations in Fig. 1 and 5, respectively. The dispersion relations for the beam modes are weakly dispersive, and we have $D\left(2 \omega_{0}, 2 k_{0}\right) \approx 0$ in terms of the dispersion relation $D(\omega, k)$, where we have $D\left(\omega_{0}, k_{0}\right)=0$. For this case we cannot expect a nonlinear Schrödinger (NLS)-type equation to account for the weakly nonlinear evolution of these waves. ${ }^{43}$ The space-time evolution of wave components with large wavenumbers that are also excited, albeit at smaller amplitudes, can be described by an NLS-equation with the assumption that the waveforms within the wavenumber spectrum are only weakly coupled so that the evolution of wavenumbers $k \gg k_{0}$ are independent of the wavenumbers $k \approx k_{0}$.

\section{E. Space-time intermittency}

The plasma fluctuations are strongly intermittent in time as well as in space, as illustrated in Fig. 8. We show here the time variation of the normalized background electron density, $n_{01} / n_{0}$, as detected at two positions. Together with the signal we show its wavelet transform. ${ }^{49}$ The wave amplitude increases exponentially in a short initial interval, saturates and then has significant variations in amplitude. The early parts of the oscillations (in the interval $t \in\{100 ; 300\}$ ) the oscillations are noticeably anharmonic, as found analytically for large amplitude electron waves with immobile ions. ${ }^{50}$ This anharmonic nature can be made evident by expanding the time axis, with a short time sample shown in Fig. 9. The positive excursions are larger than the negative ones, while the average value of the oscillations is zero. As a further evidence of the anharmonic wave-functions we note the strong harmonic generation seen in the wavelet transform. Second harmonics of the electron plasma frequency are seen at several of the large amplitude bursts, sometimes also weak signatures of the third harmonics are found. The wavelet analysis supplements the space-time Fourier transform in Fig. 6 where the localization in space and time is lost, allowing on the other hand a compact representation.

Closer inspection of short time samples as in Fig. 9 show that the dominant frequency is initially below the plasma frequency, corresponding to the beam mode. The wave amplitude grows exponentially until $t \approx 70-80$. The intensity of this wave component is reduced when the plateau has formed in the electron velocity distribution function. During this time interval, the ions are not yet set in motion. The plateau in the beam electron distribution is fully developed around $t \approx 200$. The ensuing amplitude variation seen, for instance, in Fig. 9 is partly due to the beating of different wave modes. Simultaneously, however, the ion dynamics
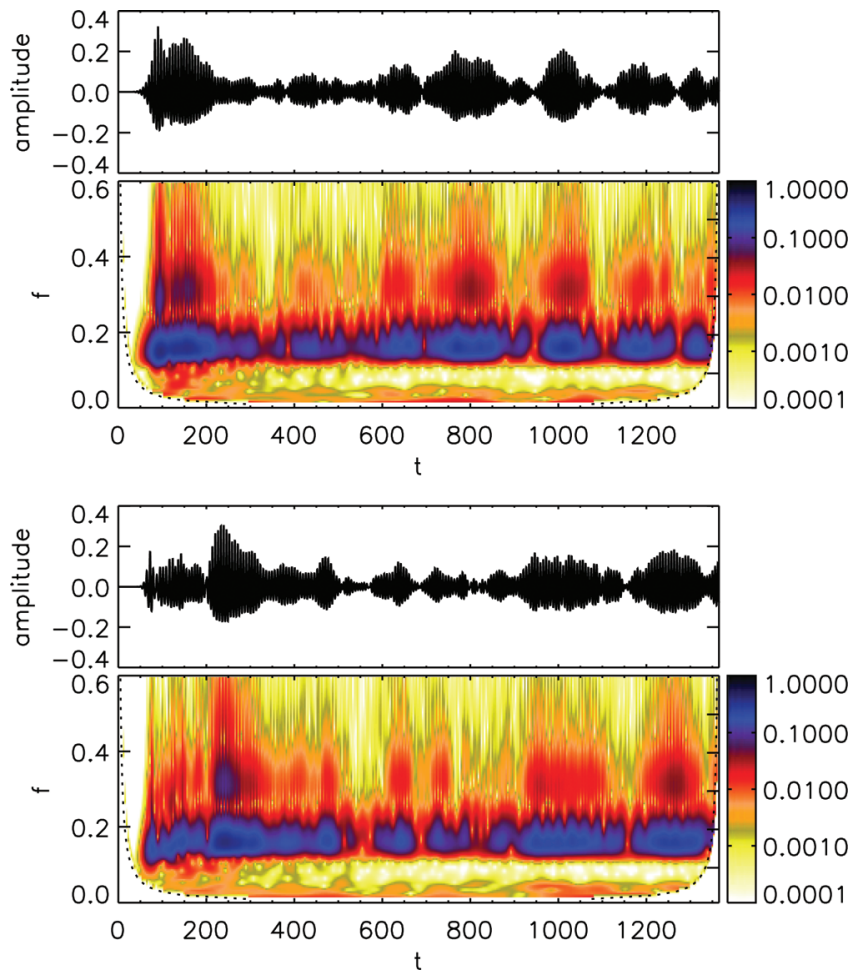

FIG. 8. (Color online) Illustration of the space-time intermittency of the oscillations, here illustrated by the normalized density variations of the background electron density. The signal is shown for two positions $2 / 5$ and $3 / 5$ of the entire length of the system. A corresponding figure shows the amplitude of a wavelet transform of the signals. The wavelet amplitude is shown on a logarithmic color-scale. The most intense frequency band corresponds to the electron plasma frequency $\omega_{p e} / 2 \pi$. Note bursts of second harmonics, and weak signatures of a third harmonic signal. The lower left and right hand corners of the wavelet transforms are omitted, since they contain edge effects.

begin to be important. The conclusion from Fig. 9 is that the electron nonlinearities (those found with immobile ions) develop in the initial phase. For times $t>200$, the waves appear as narrow band only when inspected in the time domain, where all modes have frequencies close to the electron plasma frequency. In a wavenumber presentation, many modes are excited.

At later times we observe a bursty nature of the oscillations, while at the same time a low frequency part of the signal develops. The two time signals shown in Fig. 8 are noticeably different, although they are obtained at relatively

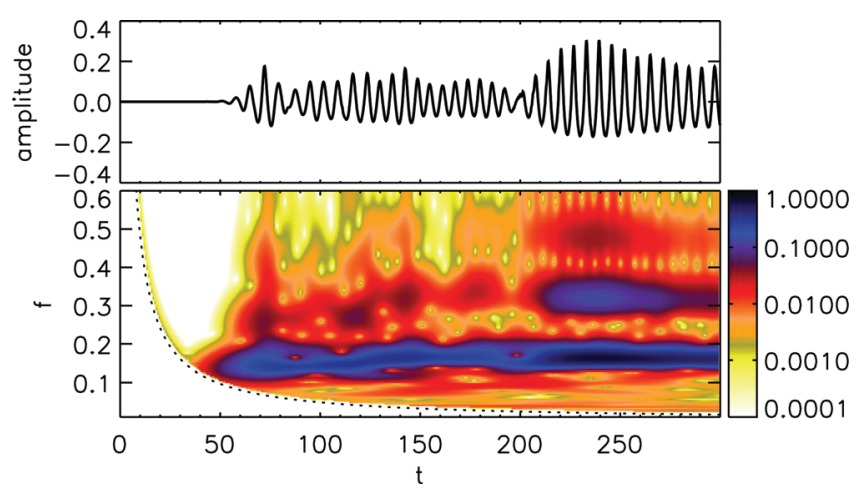

FIG. 9. (Color online) Short-time sample of a wavelet transform, see Fig. 8. 
close spatial positions, thus illustrating the spatial intermittency as well. The peak electron density perturbation becomes significant in some of the bursts, up to $n_{1} / n_{01} \approx 0.3$. The intermittent or "bursty" nature of the Langmuir wave field and the corresponding low frequency fluctuations observed in our numerical analysis is consistent with the sporadic nature of phenomena found in nature. ${ }^{1,12}$

\section{F. Summary of video}

A short video clip (plasma waves.mpg) illustrates the spatiotemporal intermittency of the waves in detail. The video is self-explanatory, with the first frame showing the electron beam, followed by the set-up for the autoscale/ fixed-scale representation of figures for the densities of ions, the background and the beam electrons. A summary is given in Fig. 10. Note that some small ion motion can be observed already from the beginning of the electron beam instability. Due to the finite electron/ion-mass ratio, ions are set into motion from the very beginning of the instability. Usually this motion would be too small to be seen, but due to the extremely low noise level in the Vlasov code, this ion motion can be seen on the autoscaled figure in the lower left corner in the video.

In the early phase of the instability the ions are affected only due to the artificial low mass ratio. The dominant signal has the form of a finite amplitude wave-packet, which is illustrated by the phase-space representations in Fig. 2. The signal has the visual appearance of a soliton. It is here seen to disintegrate due to electron trapping, see Fig. 2. The modes supported by the electron beam (right outermost panels) initially have the form of a sinusoid modulated by a near Gaussian envelope. The sinusoidal oscillations develop a near cnoidal waveform containing higher harmonics. Eventually, these beam modes develop an irregular spiky spatial variation. During this evolution, the oscillations supported by the back-
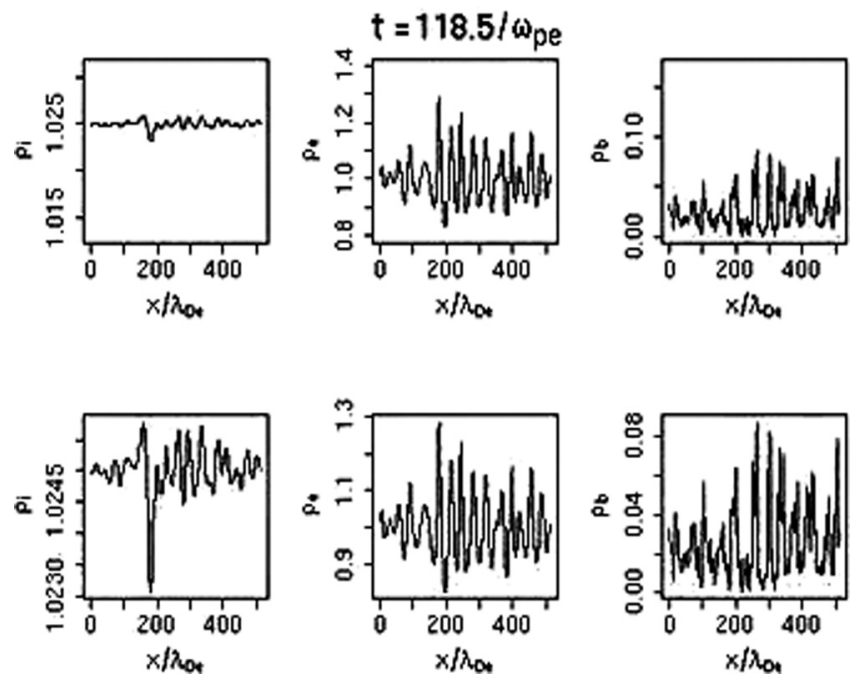

FIG. 10. Illustration of the video set-up. The top row is with fixed scales, the lower row with autoscale, allowing observation of also very small amplitudes. The left column shows the ion density, middle column shows the electron background density, and the right hand column is for the beam electron density. On the top of the figure we have time in units of $\omega_{p e}^{-1}$ (enhanced online). [URL: http://dx.doi.org/10.1063/1.3582084.1] ground electrons remain much more regular in comparison. The modes distinguished here refer to different regions of $(\omega, k)$-space. The time evolution summarized so far occurs within a time-span of $\sim 100 / \omega_{p e}$, which is so short that the ion density has only been perturbed slightly. Similar observations were made in a related laboratory experiment. ${ }^{51}$ For $t>110 / \omega_{p e}$, quasistationary density depletion (saturating at $\delta n / n \approx 0.02$ ) begins to develop in the ion density (near the position $x / \lambda_{D e} \approx 200$ ). The electron plasma waves are strongly modulated in the vicinity of this depletion, but we see no trapped wave component. This ion depletion is a fluid phenomenon, and not associated with an ion phase-space vortex.

\section{ANALYTICAL RESULTS}

The numerical results shown before demonstrate that the Langmuir, the electron acoustic as well as the beam-mode branches are excited in the system, see the dispersion relation shown in Fig. 5. The standard Zakharov set of model equations ${ }^{12,52}$ cannot account for the details in the nonlinear evolution of the observed waves. A more general set of basic nonlinear wave equations have been proposed, ${ }^{53,54}$ here written in a general three-dimensional version in the rest frame of the dense electron population. We have

$$
\begin{gathered}
-2 i \omega_{0} \frac{\partial}{\partial t} n_{1}-u_{t 1}^{2} \nabla^{2} n_{1}-\omega_{0}^{2} n_{1}+\frac{e}{m} n_{01} \nabla^{2} \phi \\
=-\frac{e}{m}\left(\frac{n_{01} / T_{e 1}}{n_{01} / T_{e 1}+n_{02} / T_{e 2}}\right) \nabla \cdot(\bar{n} \nabla \phi)
\end{gathered}
$$

for the background electrons with space-time varying density $n_{1}$, where the thermal velocity is $u_{t 1}$. To simplify the notation, we let thermal velocities include a factor 3 from here on. Both $n_{1}$ and $\phi$ in Eq. (5) vary on a time scale much slower than $\omega_{0}^{-1}$.

For the electron component forming the "plateau" in the dispersion relation shown in Fig. 5 we find

$$
\begin{gathered}
-2 i \omega_{0} \frac{\partial}{\partial t} n_{2}-u_{t 2}^{2} \nabla^{2} n_{2}-\omega_{0}^{2} n_{2}+2 i \omega_{0} \mathbf{u}_{0} \cdot \nabla n_{2} \\
-2 \mathbf{u}_{0} \cdot \nabla \frac{\partial}{\partial t} n_{2}+u_{0}^{2} \nabla^{2} n_{2}+\frac{e}{m} n_{02} \nabla^{2} \phi \\
=-\frac{e}{m}\left(\frac{n_{02} / T_{e 2}}{n_{01} / T_{e 1}+n_{02} / T_{e 2}}\right) \nabla \cdot(\bar{n} \nabla \phi)
\end{gathered}
$$

for the space-time varying density $n_{2}$ and thermal velocity $u_{t 2}$ while $u_{0}$ is the average (unperturbed) velocity of these electrons. We have a rapidly varying wave component with $\exp \left(-i \omega_{0} t\right)$ and slowly varying amplitudes $n_{j}$ for $j=1,2$. The slowly varying bulk plasma density is denoted by $\bar{n}$. We have ignored small terms like $\partial^{2} n_{j} / \partial t^{2}$ as compared to $\omega_{0} \partial n_{j} / \partial t$ for $j=1,2$. The analysis is based on the observation that the two electron species are clearly distinguished in phase-space. ${ }^{55}$ The left sides of Eqs. (5) and (6) are linear and can be derived from Eqs. (2) and (3). The different waveforms supported by the background and the plateau electrons are clearly discernible on Fig. 6. The model suggested here becomes inapplicable when the beam and background electron components become mixed. In the present simulations this mixing is a slow process, at least within the time-span of 
the simulations. As the plateau constituted by the original beam electrons is distorted at later times, the electron acoustic mode will slowly be damped by Landau damping.

The relations (5) and (6) are also here coupled through Poisson's equation (4), where $n_{1}, n_{2}$, and $\phi$ now refer to high frequency variations. If we let $u_{0}=0$ and $T_{e 1}=T_{e 2}$ with $u_{e 1}=u_{e 2}$ we recover the corresponding part of the standard Zakharov equation by adding the two Eqs. (5) and (6) and using Eq. (4).

In a fluid ion model, the evolution of the low frequency plasma density is governed by

$$
\frac{\partial^{2}}{\partial t^{2}} \bar{n}-C_{s}^{2} \nabla^{2} \bar{n}=\left(\frac{\omega_{p 0}}{\omega_{0}}\right)^{2} \frac{\varepsilon_{0}}{M} \nabla^{2}|\nabla \phi|^{2},
$$

where $\omega_{p 0}$ is the electron plasma frequency derived from the total plasma density $n_{0}=n_{01}+n_{02}$ The sound speed

$$
C_{s}=\sqrt{\frac{n_{0} T_{e 1} T_{e 2} / M}{n_{02} T_{e 1}+n_{01} T_{e 2}}} \equiv \sqrt{\frac{T_{e f}}{M}}
$$

is derived from the pressure of both the background and the plateau electrons. ${ }^{53,54}$ For later reference we introduced an effective electron temperature $T_{e f}$. In writing Eq. (7) we ignored the drift velocity of the plateau electrons, which gives a small correction of minor importance. If we linearize Eqs. (5) and (6), we recover the basic dispersion relation $\omega_{0}=\omega_{0}\left(k_{0}\right)$ in, for instance, Fig. 5 by taking $\partial / \partial t \rightarrow 0$ and $\nabla \rightarrow i \mathbf{k}_{0}$. We explicitly used the wave potential $\phi$ in the Eqs. (5-7) as a reminder of the assumption of electrostatic waves. When using the equations, it is simpler to introduce $\mathbf{E}=-\nabla \phi$.

\section{A. The relative density variations}

We found in Figs. 3 and 6 that the relative oscillation amplitudes of the background and beam electrons can be significantly different. This observation can be substantiated by linearizing Eqs. (5) and (6) to give the Fourier transformed expressions

$$
\begin{gathered}
\frac{n_{1}}{n_{01}}=\frac{e}{m} \frac{k_{0}^{2} \phi}{k_{0}^{2} u_{t 1}^{2}-\omega_{0}^{2}} \\
\frac{n_{2}}{n_{02}}=\frac{e}{m} \frac{k_{0}^{2} \phi}{k_{0}^{2} u_{t 2}^{2}-\left(\omega_{0}-\mathbf{k}_{0} \cdot \mathbf{u}_{0}\right)^{2}} .
\end{gathered}
$$

Here $\omega_{0}$ and $\mathbf{k}_{0}$ are related through the linear dispersion relation (1). We note from Eqs. (9) and (10) that for large ratios $n_{02} / n_{01}$ we can have significant differences between the fluctuation amplitudes of $n_{1}$ and $n_{2}$, in qualitative agreement with Figs. 3 and 6 . For $k_{0}=0$ and $\omega_{0}=\omega_{p e}$. we have $n_{1} / n_{01}=n_{2} / n_{02}$. For parts of the dispersion relation we find the two density perturbations, $n_{1}$ and $n_{2}$, to be in counterphase. The results are illustrated in Fig. 11, where we used Eqs. (9) and (10) and inserted the dispersion relation $\omega_{0}\left(\mathbf{k}_{0}\right)$ to display the result as a function of wavenumber $k_{0}$. We used the same parameters as in the dispersion relation in Fig. 5. For frequencies larger than the electron plasma frequency, the relative oscillation amplitude of the background

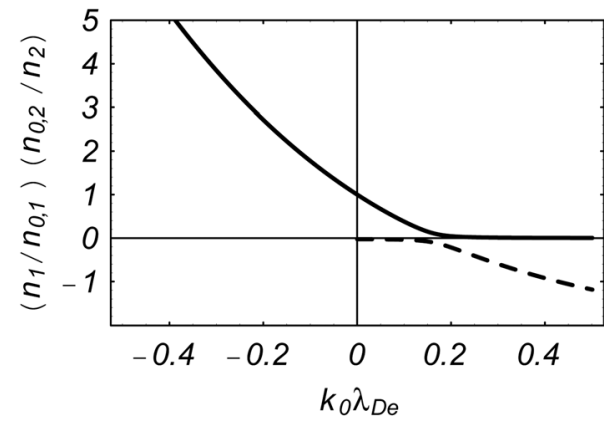

FIG. 11. Normalized relative density fluctuations $\left(n_{1} / n_{01}\right)\left(n_{02} / n_{2}\right)$ as a function of $k_{0}$ for the two branches of the dispersion relation in Fig. 5. The branch that is heavily damped by electron Landau damping is omitted here.

electron component is large, while it is relatively smaller for the electron acoustic branch and its continuation. The results of the present subsection apply for the linear phase of the oscillations, but will be used for an estimate for the weakly nonlinear wave analysis.

\section{THE OSCILLATING TWO-STREAM INSTABILITY}

First we present a simple analysis of the oscillating twostream instability ${ }^{37}$ for the present conditions. We assume $T_{e 2} \gg T_{e 1}$ and $n_{01} \gg n_{02}$ and let $\omega_{0}\left(\mathbf{k}_{0}\right)$ represent an oscillation on the electron acoustic mode, with an electric field $\mathbf{E}_{0} \exp \left(-i\left(\omega_{0} t-\mathbf{k}_{0} \cdot \mathbf{r}\right)\right)$, with $\mathbf{E}_{0}\left\|\mathbf{k}_{0}\right\| \widehat{\mathbf{x}}$. For this mode we have for the high-frequency density fluctuations that $n_{2} \gg n_{1}$. For the present unmagnetized model, the preferred direction of the initial unperturbed state is unambiguously given by $\mathbf{E}_{0}$. For the physical problem the preferred direction is given by the magnetic field aligned radar scattering, which is also the direction of the electron beam propagation. Considering only large velocities $u_{0}$, the wavenumber $k_{0}$ is small. When we perturb this primary long-wavelength wave with a small amplitude wave with wavenumber $\mathbf{K}$, the largest charge separations induced by $\mathbf{E}_{0}$ will be found when $\mathbf{K} \| \mathbf{E}_{0}$. A one-dimensional analysis taking the preferred direction along $\mathbf{E}_{0}$ will consequently capture the dominant nonlinear interactions.

We now assume that waves on the Langmuir wave like dispersion relation are excited for wavenumbers $K \gg k_{0}$ by the nonlinear evolution of the primary waves generated by the electron beam instability. On this branch we have $n_{1} \gg n_{2}$, where we use the results of Fig. 11 for an estimate. With these assumptions the sole role of the plateau electrons is to support the oscillations with frequency $\omega_{0}$.

We consider a perturbation of the initial plane wave

$$
\begin{aligned}
E(x, t)= & E_{0} e^{-i\left(\omega_{0} t-k_{0} x\right)}+E_{+} e^{-i\left(\omega_{0}+\Omega\right) t+i\left(k_{0}+K\right) x} \\
& +E_{-} e^{-i\left(\omega_{0}-\Omega\right) t+i\left(k_{0}-K\right) x},
\end{aligned}
$$

assuming $\left|E_{ \pm}\right| \ll E_{0}$. The low frequency plasma density variation is related to $E(x, t)$ by Eq. (7) with

$$
\bar{n}=\bar{n}_{+} e^{-i(\Omega t-K x)}+\bar{n}_{-} e^{+i(\Omega t-K x)} .
$$

We can determine the perturbed electric field by linearizing Eq. (5). We assume the electron beam velocity to be large compared to the background electron thermal velocity and let $k_{0} \approx 0$. We thus obtain 


$$
\left(\omega_{p e}^{2} \frac{n_{01}}{n_{0}}-\omega_{0}^{2}+K^{2} u_{t 1}^{2} \mp 2 \omega_{0} \Omega\right) E_{ \pm}=-\frac{e^{2}}{\varepsilon_{0} m} \gamma \bar{n}_{ \pm} E_{0},
$$

to be completed by an equation accounting for the ion dynamics to obtain $\bar{n}_{ \pm}$. Since we assumed the electron beam density to be small, $n_{02} \ll n_{01}$, we have $\omega_{p e}^{2} n_{01} / n_{0} \approx \omega_{p e}^{2}$. Here we introduced the abbreviation

$$
\gamma \equiv \frac{n_{01} / T_{e 1}}{n_{01} / T_{e 1}+n_{02} / T_{e 2}},
$$

i.e. $\gamma=\left(T_{e f} / T_{e 1}\right)\left(n_{01} / n_{0}\right)$.

\section{A. Fluid model for the ions}

From $|E|^{2} \approx E_{0}^{2}+E_{0}\left(E_{+} e^{-i(\Omega t-K x)}+E_{-} e^{i(\Omega t-\dot{K} x)}\right.$ $\left.+E_{+}^{*} e^{i(\Omega t-K x)}+E_{-}^{*} e^{-i(\Omega t-K x)}\right)$ and Eq. (7), we find

$$
\left(\Omega^{2}-K^{2} C_{s}^{2}\right) \bar{n}_{+}=2\left(\frac{\omega_{p e}}{\omega_{0}}\right)^{2} \frac{\varepsilon_{0}}{M} K^{2} E_{0}\left(E_{+}+E_{-}^{*}\right),
$$

where ${ }^{*}$ denotes complex conjugate. A similar expression applies for $\bar{n}_{-}$and therefore $\bar{n}_{+}=\bar{n}_{-}^{*}$.

Eliminating $E_{+}, E_{-}, \bar{n}_{+}$, and $\bar{n}_{-}$, the dispersion relation for $\Omega$ and $K$ takes the form

$$
\begin{aligned}
\frac{\Omega^{2}-K^{2} C_{s}^{2}}{\omega_{p e}^{2} n_{01} / n_{0}-\omega_{0}^{2}+K^{2} u_{t 1}^{2}} & \times\left(\left(\omega_{p e}^{2} \frac{n_{01}}{n_{0}}-\omega_{0}^{2}+K^{2} u_{t 1}^{2}\right)^{2}\right. \\
& \left.-4 \omega_{0}^{2} \Omega^{2}\right)=-2 \gamma \frac{K^{2} e^{2}}{M m}\left(\frac{\omega_{p e}}{\omega_{0}}\right)^{2} E_{0}^{2}
\end{aligned}
$$

where $C_{S}$ is given by Eq. (8). The result (13) is contained in a more formal expression presented elsewhere. ${ }^{53}$

A numerical solution of Eq. (13) is shown in Fig. 12, giving the unstable solution only. We used normalized units, where frequencies $\Omega$ (both real and imaginary) are normalized by the ion plasma frequency $\omega_{p i}$ and wavenumbers $K$ by $\omega_{p i} / C_{s}$. We introduced $W_{E} / W_{P} \equiv \gamma \varepsilon_{0} E_{0}^{2} / n_{0} M C_{s}^{2}$. We have four solutions of Eq. (13) for $\Omega$, where one is damped, one is unstable as shown, and finally two dispersive solution where $\Im\{\Omega\}=0$

The condition for the solution $\Omega=0$ (i.e., the boundary of the unstable solution in Fig. 12) is readily obtained from Eq. (13) as

$$
2 \gamma \frac{e^{2}}{M m}\left(\frac{\omega_{p e}}{\omega_{0}}\right)^{2} E_{0}^{2}=C_{s}^{2}\left(\omega_{p e}^{2} \frac{n_{01}}{n_{0}}-\omega_{0}^{2}+K^{2} u_{t 1}^{2}\right) .
$$

The right hand side is positive for all $K$ provided $\omega_{p e}^{2} n_{01} / n_{0} \geq \omega_{0}^{2}$. For the present simulation results this inequality is satisfied in general. If $\omega_{p e}^{2} n_{01} / n_{0} \geq \omega_{0}^{2}$, the oscillations of the Langmuir wave like branch are driven below their resonance frequency and the response is in phase with the pump wave. ${ }^{37}$ If the opposite inequality holds, the response is in counter-phase for a certain wavenumber interval, and the wave stability properties are changed correspondingly.

As an approximation, with $\omega_{0}^{2} \approx \omega_{p e}^{2} n_{01} / n_{0}$ and $\Im\{\Omega\} \ll C_{s} K$, we find $\Re\{\Omega\}=0$ and the growth rate in the familiar form
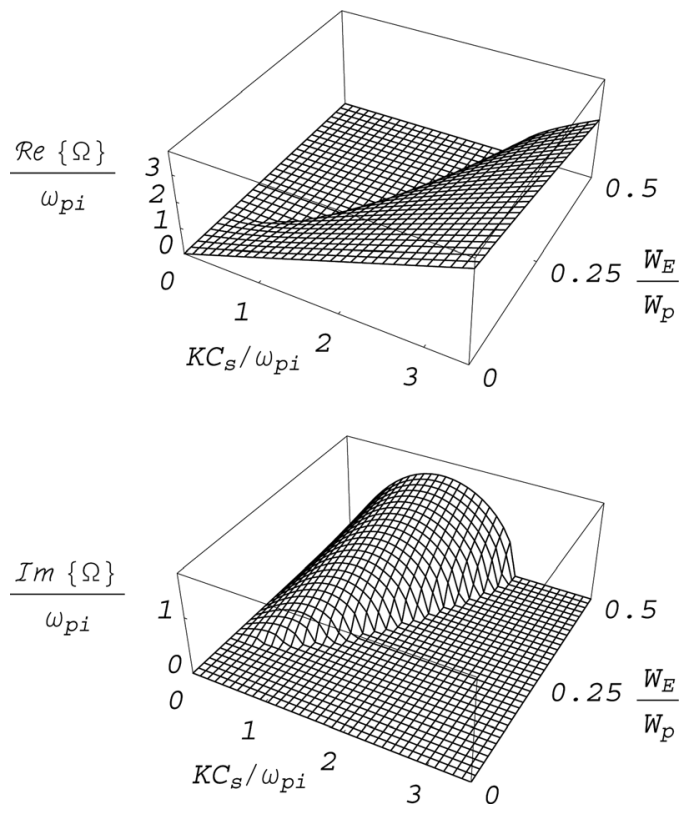

FIG. 12. Real and imaginary parts of the frequency of the unstable solution of Eq. (13). The results are shown in normalized units for the case where $\omega_{0}=0.8 \omega_{p e}$, assuming an electron-hydrogen mass ratio and $n_{02} / n_{0}=0.0025$ as in Fig. 1. Note that here $\Re\{\Omega\}=0$ where $\Im\{\Omega\} \neq 0$ We have $K C_{s} / \omega_{p i}=K \lambda_{D e} \sqrt{T_{e f} / T_{e 1}}$.

$$
\Im\{\Omega\}=\frac{K u_{t 1}}{2 \omega_{0}} \sqrt{\frac{2 \gamma}{C_{s}^{2}} \frac{e^{2} E_{0}^{2}}{M m}-K^{2} u_{t 1}^{2}} .
$$

For this case, we have an instability when

$$
K^{2} u_{t 1}^{2}<\left(2 \gamma / C_{s}^{2}\right)\left(e^{2} E_{0}^{2} / M m\right)
$$

In the simulations we can find $E \approx(m / e) u_{t h}^{2} / \lambda_{D e}$ for the present case, while $\omega_{0} / \omega_{p e} \approx 0.7-0.8$. These analytical results show the marginally unstable wavenumber to be given by $K_{m} \lambda_{D e} \approx 2$, which is somewhat larger than the observed value at saturation $K_{m} \lambda_{D e} \approx 0.5$, see, for instance, Fig. 7. The analysis refers, however, to ideal fluid conditions. Kinetic ion effects, to be discussed in Sec. V B reduce the unstable wavenumber range.

The mode $(\Re\{\Omega\}, K)$ need not satisfy any linear dispersion relation, for ion acoustic waves, for instance. The present simplified results give $\Omega$ either real or imaginary. A more detailed kinetic analysis, including $k_{0} \neq 0$, can give a small real part of $\Omega$ for the unstable conditions.

Simultaneously with the evolution of the oscillating twostream instability, wave-steepening and harmonic generation of the electron acoustic mode, corresponding to the almost linear parts of the dispersion relation, see Fig. 5, can occur.

The foregoing analysis was presented in one spatial dimension to be consistent with our numerical simulations. A generalization to two or three spatial dimensions is straightforward.

\section{B. Kinetic model for the ions}

A fluid model for the ion dynamics will be adequate when $T_{e} \gg T_{i}$, but this inequality is seldom fulfilled in 
nature. Our numerical results refer to a case where $T_{e 1}=T_{i}$. For this and similar cases, we anticipate that ion Landau damping will be important for the stable part of the low frequency oscillations. The linearly unstable branch is influenced by ion kinetic effects. In the quasineutral limit we use the linearized ion Vlasov equation for the slowly varying ion velocity distribution function $\bar{f}(x, u, t)$ with $\bar{n}=\int \bar{f} d u$ to obtain

$$
\frac{\partial}{\partial t} \bar{f}+\mathbf{u} \cdot \nabla \bar{f}-C_{s}^{2} \frac{\partial}{\partial x} \bar{n} f_{0}^{\prime}(u)=\left(\frac{\omega_{p 0}}{\omega_{0}}\right)^{2} \frac{\varepsilon_{0}}{M} f_{0}^{\prime}(u) \nabla|\nabla \phi|^{2},
$$

replacing Eq. (7). We introduced the unperturbed ion velocity distribution function as $f_{0}(u)$, normalized so that $\int_{-\infty}^{\infty} f_{0}(u) d u=1$. Models of the form (16) have been used in many studies of weakly nonlinear waves. ${ }^{4,56,57}$ For an unstable mode, $\Im\{\Omega\}>0$, the spatially one-dimensional model

$$
\bar{n}(\Omega, K)=2 \frac{\varepsilon_{0}}{M C_{s}^{2}}\left(\frac{\omega_{p e}}{\omega_{0}}\right)^{2} E_{0}\left|E_{+}+E_{-}^{*}\right| \frac{G(\Omega / K)}{1-G(\Omega / K)},
$$

is replacing Eq. (12). We here introduced

$$
\left.G(\Omega / K) \equiv C_{s}^{2}\right\}_{-\infty}^{\infty} \frac{f_{0}^{\prime}(u)}{u-\Omega / K} d u,
$$

with the Landau contour of integration running below the singularity when $K>0$ as indicated. If we insert $f_{0}=\delta(u)$ in Eq. (17), we reproduce the fluid result with cold ions. When $f_{0}(u)$ is a finite temperature Maxwellian, we have $G(\Omega / K)=\frac{1}{2}\left(T_{e f} / T_{i}\right) Z^{\prime}(\Omega / K)$ in terms of the derivative of the plasma dispersion function. ${ }^{37}$ At $\Omega=0$, we have $G(0)=-T_{e f} / T_{i}$, and for $T_{e f} \gg T_{i}$, we find that Eq. (17) inserted into Eq. (11) reproduces Eq. (14) found from a cold ion fluid model. We can thus argue that the simple ion fluid model will reproduce the basic properties of the instability, with kinetic effects being relevant only for details in the growth rates. As far as the $\Omega=0$ reference value of the electric field Eq. (14) is concerned, the ion kinetic effects give a factor of approximately $\left(T_{i}+T_{e f}\right) / T_{e f}$. This correction amounts to approximately a factor 2 for the present simulation conditions.

The kinetic ion model summarized in the present subsection can be particularly relevant for non-Maxwellian ion distributions often found in the ionosphere, but we anticipate that these cases will require a numerical solution of the resulting (implicit) dispersion relation. The propagating ion sound modes will be strongly Landau damped when $T_{e} \approx T_{i}$ and these waves will have a correspondingly weak signature in the $(\omega, k)$ representation.

\section{DISCUSSIONS AND CONCLUSIONS}

We have analyzed the electron density variations of the initial $\sim 100$ electron plasma periods of the simulations and find here only activity on the low-frequency electron acoustic-like branch of the dispersion relation, see Fig. 1. The real and imaginary parts agree well with the numerical result, as shown by open and full circles in Fig. 1 for the initial linear phase of the instability. In particular, we recall that the analytical result was obtained by a simplified water-bag model rather than a full kinetic dispersion model. ${ }^{36}$ The large linewidths in Fig. 3 reflect the growth-rate of the linear instability. Simultaneously, we find a strong scattering in velocity space of the beam electrons. The activity in the ion density is negligible in the same time-interval. As the linear instability saturates, the high frequency (Langmuir-like) part of the dispersion relation becomes populated, see Fig. 3. Investigations of the ion density at later times show first the evolution of a long-wavelength spatially varying density, which is almost stationary, and does not follow any linear dispersion relation. Later, we find a slow evolution of a backward traveling ion acoustic wave and a smaller amplitude forward propagating component, which is barely noticeable in Fig. 7. The latter component originates from the decay from the beam modes to the ion sound mode and a backward traveling electron plasma wave.

If the numerical simulation should be extended for times longer than those considered in the present study, we believe that collisions should be taken into account: for the ionospheric regions of interest here, the plasma parameter (number of particles in a Debye sphere) has typical values of the order of $N_{p} \approx 10^{4}$. We can use the estimate for the electron collision time $\tau_{c} \approx N_{p} / \omega_{p e}$ and find this to be approximately 250 ion plasma periods. For longer time-spans the electron plateau will be eroded by collisions and the electron acoustic mode will become heavily damped and unimportant for the wave dynamics.

In order to illustrate the dynamics at a late time (as measured in units of the ion plasma period), we also performed simulations with extended time durations, up to $1300 / \omega_{p e}$, and a reduced mass ratio $M / m=200$. We find an enhanced activity both in the long and short wavelength parts of the ion sound spectrum.

We have studied the electron and ion phase-spaces at selected time intervals. Electron holes can be observed at several stages of the instability. Although we note a strong activity also in ion phase-space, we find it interesting that no long-lived ion holes are formed. Numerical results ${ }^{20}$ have indeed indicated that no ion holes should be formed for the present temperature ratios $T_{e} / T_{i}$. However, these observations refer to a case without electron plasma wave activity. In studies of ion upflow and naturally enhanced ion lines, ${ }^{58}$ it was found that NEIALs were found predominantly at enhanced electron-ion temperature ratios. Under these conditions it is possible that ion and electron holes can have a more important role than in our simulations. It will be worthwhile to repeat simulations like ours for these conditions, taking into account also the nonthermal ion velocity distribution due to the ion outflow.

We have presented results from numerical solutions of the coupled electron-ion Vlasov equations, using parameters that are relevant also for ionospheric conditions but nonetheless differ from those that can be modeled by standard studies based on, for instance, Zakharov-type equations with energy sources and sinks. ${ }^{12}$

Our numerical results show the following features relevant also for the observations of NEIALs: (1) The oscillating two-stream instability ${ }^{37}$ of the electron beam generated 
waves excite high frequency as well as low frequency oscillations. The latter do not necessarily follow the ion acoustic dispersion relation and can appear as a broad unshifted or a weakly shifted line, filling-in between the two normally occurring ion acoustic lines. (2) The oscillating two-stream instability simultaneously excites electron plasma waves with $\omega \approx \omega_{p e}$ in a broad wavenumber range, that can decay to another electron wave and an ion sound wave. An important point is that the electron acoustic mode can participate in the decay and the Langmuir-condensate consequently becomes leaky when this line of decay is active. ${ }^{53} \mathrm{~A}$ part of the decay product is an asymmetric population of sound waves propagating in opposite directions. For $T_{e} \approx T_{i}$ these waves are heavily ion-Landau damped. (3) Early in the evolution of the Langmuir waves, we find nonlinear "spiky" waveforms developing, a feature not involving the ion dynamics. These effects are not included in the standard models based on the Zakharov equations. (4) A robust feature of our simulation is the presence of a wave branch with very small propagation velocities, smaller than the ion sound speed. These are excited in a range of wavenumbers $K$, with an estimate $0 \leq|K| \leq u_{t 1}^{-1} \sqrt{\left(2 \gamma / C_{s}^{2}\right)\left(e^{2} E_{0}^{2} / M m\right)}$, see Secs. $\mathrm{V} A$ and $\mathrm{V} \mathrm{B}$. Within this range it will be possible to observe both Langmuir and weakly shifted ion lines simultaneously. This analysis is, however, based on the presence of an electron acoustic branch, and this will disappear at later times. Propagating ion acoustic waves appear late in our simulations. An ion flow will make the unshifted line appear as propagating, but we need sonic flows to confuse it with the ion-line, and this will be exceptional. (5) Late in the evolution of the waveforms, we find that electron phase-space vortices (but no ion vortices) are formed. These can saturate in nonlinear ion-acoustic pulses, propagating at a speed larger than the linear ion sound speed. ${ }^{16,59}$ Many of these electron holes are quite faint (i.e., having relative density perturbations $\ll 1$ ). Of those macroscopically noticeable (with density variations exceeding $\sim 2 \%$ ), we have approximately a density of one electron hole per $\sim 300 \lambda_{D e}$, with an individual lifetime of approximately 200 electron plasma periods, $\tau_{p e}$. These values refer to $\alpha=0.1$. The electron holes are thus transient phenomena here, but they can be formed by electron trapping at any time of the saturated stage of the instability. Very large electron holes are formed during the first phase of the linear instability, where the initial electron beam is dispersed, but these holes disperse within the first 50 $\tau_{p e}$. It is possible that the enhanced temperature ratios $T_{e} / T_{i} \geq 2$ observed in some experiments ${ }^{58}$ will allow longlived ion phase-space vortices.

A limitation of our simulations is the short spatial domain, which does not allow us to study influences of weak large scale density gradients. Other studies ${ }^{17,18}$ indicated that these effects could be important. Those results rely on an entirely different computational mode ${ }^{60}$ based on the weak turbulence approximation. We have studied more localized density gradients in ion density, but these were of scale lengths too short to be relevant for those previous studies, ${ }^{17,18}$ and no conclusive results can be presented.

Finally, we emphasize that our results have applications beyond their relevance for the ionospheric observations and apply for electrostatic plasma waves generated by low density cold electron beams in collisionless plasmas. The conditions analyzed in the present work can be found in many laboratory plasmas as well as in nature.

\section{ACKNOWLEDGMENTS}

The work was supported in part by a grant from Uppsala University, Department of Physics and Astronomy and in part by the Norwegian National Science Foundation. One of the authors (HLP) thanks Eduard Kontar and Patrick Guio for valuable discussions.

${ }^{1}$ F. Sedgemore-Schulthess and J-P. St.-Maurice, Surv. Geophys. 22, 55 (2001).

${ }^{2}$ G. Bekefi, Radiation Processes in Plasmas (John Wiley \& Sons, New York, 1966)

${ }^{3}$ S. C. Buchert, A.P. van Eyken, T. Ogawa, and S. Watanabe, Adv. Space Res. 23, 1699 (1999).

${ }^{4}$ T. Grydeland, C. La Hoz, T. Hagfors, E. M. Blixt, S. Saito, A. Strømme, and A. Brekke, Geophys. Res. Lett. 30, 1338, doi:10.1029/2002GL016362 (2003).

${ }^{5}$ M. T. Rietveld, B. Isham, T. Grydeland, C. La Hoz, T. B. Leyser, F. Honary, H. Ueda, M. Kosch, and T. Hagfors, Adv. Space Res. 29, 1363 (2002).

${ }^{6}$ M. T. Rietveld, P. N. Collis, and J-P. St-Maurice, J. Geophys. Res. 96, 19291, doi:10.1029/91JA01188 (1991).

${ }^{7}$ J. E. Wahlund, F. R. E. Forme, H. J. Opgenoorth, M. A. L. Persson, E. V. Mishin, and A. S. Volokitin, Geophys. Res. Lett. 19, 1919, doi:10.1029/ 92GL02101 (1992).

${ }^{8}$ K. B. Dysthe, E. Leer, J. Trulsen, and L. Stenflo, J. Geophys. Res. 82, 717, doi:10.1029/JA082i004p00717 (1977).

${ }^{9}$ J. A. Fejer, Geophys. Res. Lett. 4, 289, doi:10.1029/GL004i007p00289 (1977).

${ }^{10}$ F. Forme, Ann. Geophys. 17, 1172 (1999).

${ }^{11}$ F. Forme, Y. Ogawa, and S. C. Buchert, J. Geophys. Res. 106, 21503, doi:10.1029/2000JA900164 (2001).

${ }^{12}$ P. Guio and F. Forme, Phys. Plasmas 13, 122902 (2006).

${ }^{13}$ A. Strømme, V. Belyey, T. Grydeland, C. La Hoz, U. P. Løvhaug, and B. Isham, Geophys. Res. Lett. 32, L05103, doi:10.1029/2004GL020239 (2005).

${ }^{14}$ N. G. van Kampen and B. U. Felderhof, Theoretical Methods in Plasma Physics (North Holland Publishing Company, Amsterdam, 1967).

${ }^{15}$ I. B. Bernstein, J. M. Greene, and M. D. Kruskal, Phys. Rev. 108, 546 (1957).

${ }^{16}$ H. Schamel, Phys. Rep. 140, 161 (1986).

${ }^{17}$ E. P. Kontar and H. L. Pécseli, Phys. Rev. E 65, 066408 (2002).

${ }^{18}$ E. P. Kontar and H. L. Pécseli, Geophys. Res. Lett. 32, L05110, doi: 10.1029/2004GL022182 (2005).

${ }^{19}$ D. Fontaine, Plasma Sources Sci. Technol. 11, A113 (2002).

${ }^{20}$ H. L. Pécseli, J. Trulsen, and R. Armstrong, Phys. Scr. 29, 241 (1984)

${ }^{21}$ L. K. S. Daldorff, P. Guio, S. Børve, H. L. Pécseli, and J. Trulsen, Europhys. Lett. 54, 161 (2001).

${ }^{22}$ P. K. Shukla and B. Eliasson, JETP Lett. 77, 647 (2003).

${ }^{23}$ K. Saeki, P. Michelsen, H. L. Pécseli, and J. Juul Rasmussen, Phys. Rev. Lett. 42, 501 (1979).

${ }^{24}$ J. P. Lynov, P. Michelsen, H. L. Pécseli, J. Juul Rasmussen, K. Saeki, and V. Turikov, Phys. Scr. 20, 328 (1979).

${ }^{25}$ J. P. Lynov, P. Michelsen, H. L. Pécseli, J. Juul Rasmussen, and H. Sørensen, Phys. Scr. 31, 596 (1985).

${ }^{26}$ P. Guio, S. Børve, L. K. S. Daldorff, J. P. Lynov, P. Michelsen, H. L. Pécseli, J. J. Rasmussen, K. Saeki, and J. Trulsen, Nonlinear Processes Geophys. 10, 75 (2003).

${ }^{27}$ D. Schriver,M. Ashour-Abdalla, V. Sotnikov, P. Hellinger, V. Fiala, R. Bingham, and A. Mangeney, J. Geophys. Res. 105, 12919, doi:10.1029/ 1999JA000301 (2000).

${ }^{28}$ Y. Kasaba, H. Matsumoto, and Y. Omura, J. Geophys. Res. 106, 18693, doi:10.1029/2000JA000329 (2001).

${ }^{29}$ T. Umeda, Y. Omura, P. H. Yoon, R. Gaelzer, and H. Matsumoto, Phys. Plasmas 10, 382 (2003).

${ }^{30}$ R. A. Sprague and J. A. Fejer, J. Geophys. Res. 100, 23959, doi:10.1029/ 95JA01821 (1995).

${ }^{31}$ D. Henry and J. P. Treguier, Phys. Lett. 38A, 115 (1972). 
${ }^{32}$ Y. Kawai, Y. Nakamura, T. Itoh, T. Hara, and T. Kawabe, J. Phys. Soc. Jpn. 38, 876 (1975).

${ }^{33}$ L. K. S. Daldorff, H. L. Pécseli, and J. Trulsen, Geophys. Res. Lett. 34, L18101, doi:10.1029/2007GL031513 (2007).

${ }^{34}$ R. C. Davidson, Methods in Nonlinear Plasma Theory (Pure and Applied Physics) (Academic, New York, 1972), Vol. 37.

${ }^{35}$ R. R. Parker and A. L. Throop, Phys. Rev. Lett. 31, 1549 (1973).

${ }^{36}$ T. M. O'Neil, and J. H. Malmberg, Phys. Fluids 11, 1754 (1968).

${ }^{37}$ F. F. Chen, Introduction to Plasma Physics and Controlled Fusion, 2nd ed. (Plenum, New York, 1984), Vol. 1.

${ }^{38}$ B. Eliasson, J. Sci. Comput. 16, 1 (2001).

${ }^{39}$ B. Eliasson, J. Comp. Phys. 181, 98 (2002).

${ }^{40}$ B. Eliasson, J. Comp. Phys. 190, 502 (2003).

${ }^{41}$ S. G. Thornhill and D. ter Haar, Phys. Rep. 43, 43 (1978).

${ }^{42}$ G. B. Whitham, Linear and Nonlinear Waves (John Wiley \& Sons, New York, 1974),

${ }^{43}$ H. L. Pécseli, IEEE Trans. Plasma Sci. PS-13, 53 (1985).

${ }^{44}$ B. H. Quon, A. Y. Wong, and B. H. Ripin, Phys. Rev. Lett. 32, 406 (1974).

${ }^{45}$ P. H. Yoon, R. Gaelzer, T. Umeda, Y. Omura, and H. Matsumoto, Phys. Plasmas 10, 364 (2003).

${ }^{46}$ R. Gaelzer, P. H. Yoon, T. Umeda, Y. Omura, and H. Matsumoto, Phys. Plasmas 10, 373 (2003).
${ }^{47}$ H. L. Pécseli and J. Trulsen, Plasma Phys. Controlled Fusion 35, 1701 (1993).

${ }^{48}$ M. V. Goldman, F. Crary, D. L. Newman, and M. Oppenheim, Phys. Plasmas 7, 1732 (2000).

${ }^{49}$ S. Mallat, A Wavelet Tour of Signal Processing (Academic, San Diego, CA, 1998).

${ }^{50}$ R. W. C. Davidson and P. P. J. M. Schram, Nucl. Fusion 8, 183 (1968).

${ }^{51}$ T. Intrator, C. Chan, N. Hershkowitz, and D. Diebold, Phys. Rev. Lett. 53, 1233 (1984).

${ }^{52}$ V. E. Zakharov, Zh. Eksp. Teor. Fiz. 62, 1745 (1972);See also Sov. Phys. JETP 35, 908 (1972).

${ }^{53}$ A. Hanssen, H. L. Pécseli, L. Stenflo, and J. Trulsen, J. Plasma Phys. 51, 423 (1994).

${ }^{54}$ O. Skjæraasen, B. Krane, H. L. Pécseli, L. Stenflo, and J. Trulsen, Phys. Scr. T63, 34 (1996).

${ }^{55}$ M. Y. Yu and H. Q. Lu, Phys. Plasmas 15, 024504 (2008).

${ }^{56}$ Y. H. Ichikawa and T. Taniuti, J. Phys. Soc. Jpn. 34, 513 (1973).

${ }^{57}$ K. B. Dysthe and H. L. Pécseli, Plasma Phys. 19, 931 (1977).

${ }^{58}$ Y. Ogawa, S. C. Buchert, I. Haggstrom, M. T. Rietveld, R. Fujii, S. Nozawa, and H. Miyaoka, J. Geophys. Res. 116, A03313, doi:10.1029/ 2010JA015827 (2011).

${ }^{59}$ K. Saeki and H. Genma, Phys. Rev. Lett. 80, 1224 (1998).

${ }^{60}$ E. P. Kontar, Comput. Phys. Commun. 138, 222 (2001). 